\title{
Neuroprotection of Fasting Mimicking Diet on MPTP-Induced Parkinson's Disease Mice via Gut Microbiota and Metabolites
}

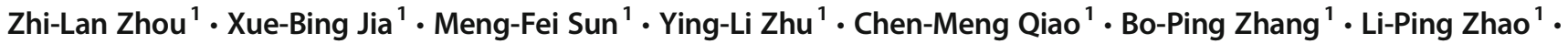 \\ Qin Yang ${ }^{1} \cdot$ Chun Cui $^{1} \cdot$ Xue Chen $^{1} \cdot$ Yan-Qin Shen ${ }^{1} \mathbb{D}$
}

Published online: 27 February 2019

(C) The American Society for Experimental NeuroTherapeutics, Inc. 2019

\begin{abstract}
Parkinson's disease (PD) is strongly associated with life style, especially dietary habits, which have gained attention as disease modifiers. Here, we report a fasting mimicking diet (FMD), fasting 3 days followed by 4 days of refeeding for three 1-week cycles, which accelerated the retention of motor function and attenuated the loss of dopaminergic neurons in the substantia nigra in 1-methyl-4-phenyl-1,2,3,6-tetrathydropyridine (MPTP)-induced PD mice. Levels of brain-derived neurotrophic factor (BDNF), known to promote the survival of dopaminergic neurons, were increased in PD mice after FMD, suggesting an involvement of BDNF in FMD-mediated neuroprotection. Furthermore, FMD decreased the number of glial cells as well as the release of TNF- $\alpha$ and IL-1 $\beta$ in PD mice, showing that FMD also inhibited neuro-inflammation. 16S and 18S rRNA sequencing of fecal microbiota showed that FMD treatment modulated the shifts in gut microbiota composition, including higher abundance of Firmicutes, Tenericutes, and Opisthokonta and lower abundance of Proteobacteria at the phylum level in PD mice. Gas chromatography-mass spectrometry and liquid chromatography-mass spectrometry revealed that FMD modulated the MPTP-induced lower propionic acid and isobutyric acid, and higher butyric acid and valeric acid and other metabolites. Transplantation of fecal microbiota, from normal mice with FMD treatment to antibiotic-pretreated PD mice increased dopamine levels in the recipient PD mice, suggesting that gut microbiota contributed to the neuroprotection of FMD for PD. These findings demonstrate that FMD can be a new means of preventing and treating PD through promoting a favorable gut microbiota composition and metabolites.
\end{abstract}

Key Words Parkinson's disease $\cdot$ fasting mimicking diet $\cdot$ gut microbiota $\cdot$ metabolites $\cdot$ neuro-inflammation $\cdot$ BDNF

\section{Introduction}

Parkinson's disease (PD) is the second most common neurodegenerative disorder and affects $2-3 \%$ of the population over 65 years of age [1]. PD is clinically characterized by motor symptoms, including rigidity, resting tremor, and bradykinesia that result from a selective loss of dopaminergic neurons in the substantia nigra pars compacta [2]. There is also a wide spectrum of nonmotor manifestations involving, for example, gastrointestinal dysfunction, including constipation and

Yan-Qin Shen

shenyanqin@jiangnan.edu.cn

1 Public Health Research Center at Jiangnan University, Wuxi Medical School, Jiangnan University, Wuxi 214122, China defecatory dysfunction, the latter potentially reflecting a presymptomatic stage of PD [3]. With less than $10 \%$ of all cases displaying a positive family history, the majority are considered sporadic [4]. Therefore, in addition to a genetic predisposition, a variety of other factors are associated with the development of PD. Animal studies show that diet-induced obesity in mice aggravates MPTP-induced dopaminergic neuron degeneration in the substantia nigra pars compacta $[5,6]$.

Dietary intervention is associated with many diseases. For example, caloric restriction has been shown to lessen the severity of neurochemical deficits and motor dysfunction in a primate model of PD [7], and intermittent fasting has been shown to protect neurons against dysfunction and degeneration in rodent models of Huntington's disease [8], but the mechanism for these benefits is largely unclear. Fasting mimicking diet (FMD) consists of three 1-week cycles, with each cycle consisting of FMD for three consecutive days separated by 
four consecutive days of ad libitum (AL) feeding [9]. FMD causes changes in markers associated with stress resistance and longevity that are similar to those caused by fasting [10], but minimizes the burden of fasting. In a pilot clinical trial (control $n=19$, FMD $n=19$ ), three cycles of FMD were selected to change the markers of aging and cell protection, such as reduce IGF-I, increase IGFBP-1, reduce glucose, increase ketone bodies, and change the cardiovascular disease risk factors (reduce C-reactive protein levels) [10]. Similarly, in another pilot clinical trial (control $n=43$, FMD $n=39$ ), three cycles of FMD decreased risk factors for aging, diabetes, cardiovascular disease, and cancer without major adverse effects [11], providing support for the use of FMD to promote health and extend lifespan. Specifically, FMD is effective in promoting lineage reprogramming and insulin generation in pancreatic islet cells from healthy $(n=5)$ and T1D $(n=5)$ subjects, which were treated with serum from subjects enrolled in a clinical trial [12]. Thus, how FMD affects PD is worth to study.

As is well-known, dietary intervention, such as everyother-day fasting, fasting for 72 and $96 \mathrm{~h}$, time-restricted feeding, alters the gut microbiota composition [13-16]. Major changes in microbiota composition can directly change microbial products, such as short-chain fatty acids (SCFAs), trimethylamine $\mathrm{N}$-oxide, tryptophan, and tyrosine derivatives [17]. The impact of the gut microbiota on the brain is profound and has been recognized to affect behavior, neurotransmitter production, microglial activity, neurogenesis, and blood-brain barrier integrity [18]. Recently, correlative evidence reveals that gut microbiota plays important roles in neuroprotection in PD mice [19-22].

In the current study, mice were treated with MPTP, to induce acute PD model, then placed on an FMD regimen. FMD can decrease the degeneration of dopaminergic neurons in the substantia nigra (SN) and lessen motor dysfunction. To investigate whether the FMD-induced microbial shift directly contributes to amelioration of $\mathrm{PD}$, microbiota from the normal saline (NS) ad libitum group and normal saline-fasting mimicking diet group were transplanted to antibiotic-pretreated PD mice respectively. Evidence suggests that FMD ameliorates PD, including reduction of striatal dopamine (DA) depletion and prevention of motor dysfunction, probably via a diet-gut microbiota-brain axis.

\section{Methods}

\section{Animals and Group Design}

Six-week-old male C57BL/6J mice (18 $\pm 2 \mathrm{~g}$ ) were acquired from the Zhaoyan New Drug Research Center (Suzhou, China) and acclimatized for 1 week before treatment. The mice were maintained ( $12 \mathrm{~h} \mathrm{light/dark}$ cycle) under pathogen-free conditions at $24 \pm 2.0{ }^{\circ} \mathrm{C}$ and $55 \pm 10 \%$ humidity and allowed free access to water and standard rodent chow food (four mice/cage) or the fasting mimicking diet (one mouse/cage). All experimental procedures were approved by the Animal Ethics Committee of Jiangnan University.

To verify the neuroprotection of FMD on PD mice, at 7 weeks of age, $\mathrm{C} 57 \mathrm{BL} / 6 \mathrm{~J}$ mice were randomly divided into four groups: the NS-AL group, which was treated with normal saline by intraperitoneal injection and fed ad libitum; the NSFMD group, which was treated with normal saline by intraperitoneal injection and fasting mimicking diet; the MPTP-AL group, which was treated with MPTP and ad libitum; and the MPTP-FMD group, which was treated with MPTP and fasting mimicking diet. In a word, mice in this experiment treated with normal saline or MPTP on the last day of the second cycle (on day 21 ; Fig. 1a). Behavior training was conducted once per day for three consecutive days (on days 26, 27, 28; Fig. 1a), and behavior test was conducted on the day after the last treatment (on day 29; Fig. 1a). The whole brain, striatum and feces were collected after behavior test (on day 29; Fig. 1a).

To explore the role of gut microbiota between PD and FMD, mice, pretreated with an antibiotic cocktail and MPTP, were randomly divided into four groups: the PD$\mathrm{PBS} / \mathrm{G}$ group, which was treated with $20 \%$ glycerol in sterile phosphate-buffered solution (PBS) by gastric gavage; the PDNA group, which was treated with gut microbiota from NSAL mice by gastric gavage; the PD-NF group, which was given gut microbiota from NS-FMD mice by gastric gavage; the PD-NF/HK group, which was treated with heat-killed (HK) gut microbiota from NS-FMD mice by gastric gavage. The striatum was collected on the day after the last treatment (on day 25; Fig. 7a). In all of these four groups, levels of striatal DA and serotonin (5-HT) were measured by highperformance liquid chromatography.

\section{FMD Treatment}

Mice were fed ad libitum with irradiated LAD0011 rodent chow (Trophic Animal Feed High-tech Co. Ltd., Jiangsu, China), containing $3.4 \mathrm{kcal} / \mathrm{g}$ of digestible energy (protein $22 \%$, carbohydrate $65 \%$, fat $13 \%$ ). On average, mice in the control group consumed $12 \mathrm{kcal} /$ day (or $3.5 \mathrm{~g} /$ day) based on pre-experiments. The experimental FMD was based on a nutritional screen that identified ingredients that allowed high nourishment during periods of low calorie consumption. The FMD consisted of two different components designated as the day 1 diet and day 2-3 diet that were fed in this respective order. The day 1 diet contained $50 \%$ of the standard daily calorie intake and was designed to adapt the mouse to a period of low caloric intake. The day 2-3 diet contained $10 \%$ of the standard daily calorie intake. All mice were supplied with fresh food during the morning hours ( $8 \mathrm{am}-10 \mathrm{am})$. Mice consumed all the supplied food on each day of the FMD regimen and showed no signs of food aversion. After the end of the 3-day FMD, we supplied 

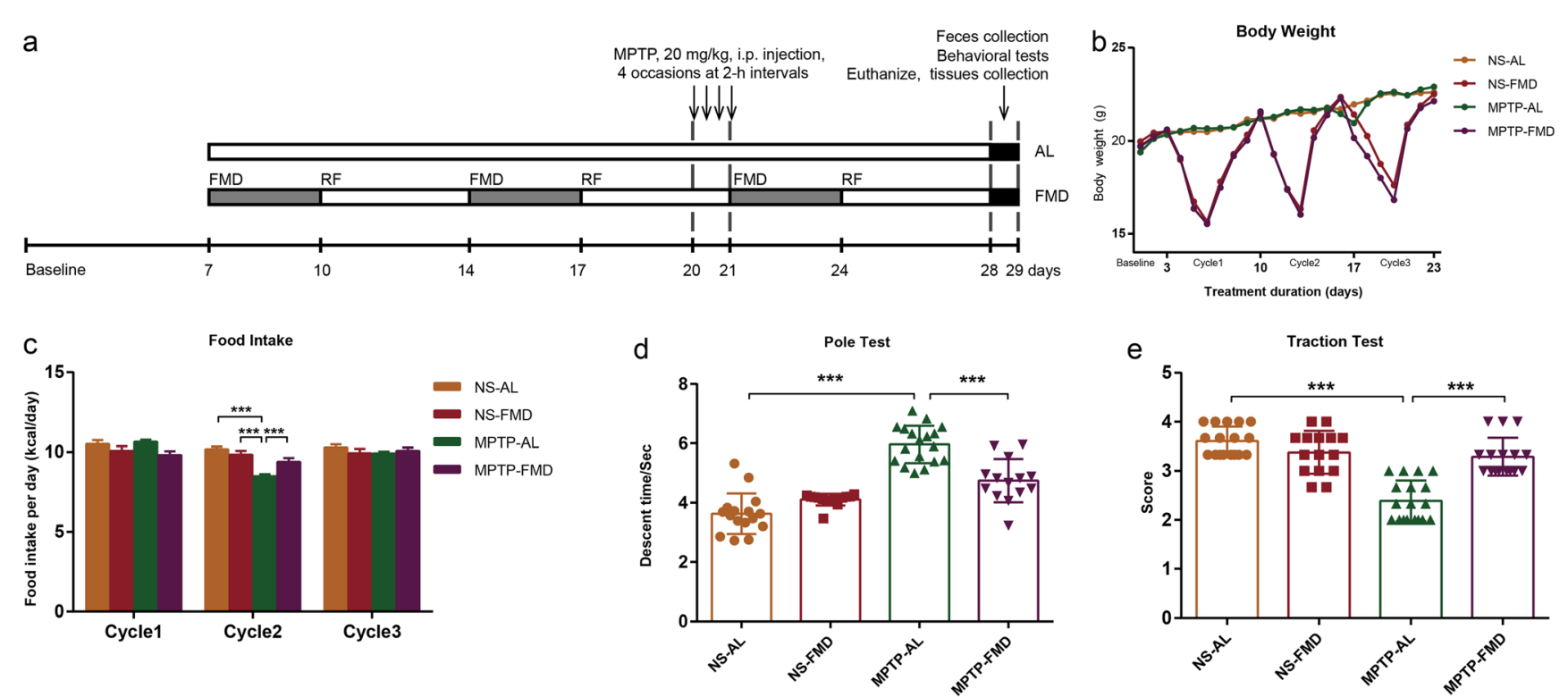

Fig. 1 FMD treatment retains motor function in PD mice without an overall reduction in calorie intake. (a) Timeline for the experimental procedure, including FMD, MPTP administration, and tissue collection. (b) Body weight changes. NS-AL group $(n=17)$, NS-FMD group $(n=$ 15), MPTP-AL group ( $n=18)$, MPTP-FMD group $(n=16)$. (c) Average food intake in per cycle, $n=13$ mice per group. (d) Pole descent test: time taken represents degree of bradykinesia, $F_{3,59}=48.325, p<0.001$. NS-

LAD0011 rodent chow ad libitum for 4 days of refeeding before starting another FMD cycle. Prior to supplying the FMD, animals were transferred into fresh cages to avoid feeding on residual chow and coprophagy. Food intake was measured daily.

\section{Antibiotic Treatment}

Antibiotic-pretreated animals were provided a cocktail consisting of $1 \mathrm{mg} / \mathrm{ml}$ bacitracin, $0.5 \mathrm{mg} / \mathrm{ml}$ gentamycin, $0.2 \mathrm{mg} / \mathrm{ml}$ ciprofloxacin, $1 \mathrm{mg} / \mathrm{ml}$ neomycin, $1 \mathrm{mg} / \mathrm{ml} \mathrm{peni-}$ cillin, $1 \mathrm{mg} / \mathrm{ml}$ metronidazole, $0.5 \mathrm{mg} / \mathrm{ml}$ ceftazidime, $0.5 \mathrm{mg} / \mathrm{ml}$ vancomycin, and $2 \mathrm{mg} / \mathrm{ml}$ streptomycin [23-25] and were administered daily by gastric gavage at a dose of $200 \mu \mathrm{l}$ [26]. Antibiotics were administered daily for 7 consecutive days prior to MPTP treatment and fecal microbiota transplantation (Fig. 7a). Two days after cessation of antibiotic treatment, mice were administered MPTP [24].

\section{MPTP Treatment}

MPTP is known to cause a destruction of the dopaminergic nigrostriatal pathway and elicit symptoms of PD [27]. C57BL/6J mice were administered intraperitoneal with MPTP (20 mg/kg dissolved in sterilized saline) in a volume of $10 \mathrm{ml} /$ $\mathrm{kg}$ of body weight on four occasions, with each administration separated by a 2-h interval [28]. In experiments involving FMD, MPTP was administered on the last day of the second FMD cycle (Fig. 1a). The control mice were treated with NS.
AL group $(n=16)$, NS-FMD group $(n=15)$, MPTP-AL group $(n=18)$, MPTP-FMD group $(n=14)$. (e) Traction test: suspension reflex score for evaluation of muscle strength and equilibrium, $F_{3,62}=33.929, p<0.001$. NS-AL group $(n=17)$, NS-FMD group $(n=15)$, MPTP-AL group $(n=$ 18), MPTP-FMD group $(n=16)$. Statistical comparison by one-way ANOVA with post hoc comparisons of LSD; data represent the means \pm $\mathrm{SEM} ; * * * p<0.001$

\section{Transplantation of Fecal Microbiota}

Fresh fecal pellets were collected from NS-AL and NS-FMD mice, then diluted immediately with sterile PBS (1 fecal pellet/ $\mathrm{ml})$. For each experiment, several fecal pellets from different mice in the corresponding group were resuspended together in PBS [24]. Briefly, the feces were steeped in sterile PBS for about $15 \mathrm{~min}$, shaken, and then centrifuged at $1000 \times \mathrm{g}, 4^{\circ} \mathrm{C}$ for $5 \mathrm{~min}$. The suspension was centrifuged at $8000 \times \mathrm{g}, 4^{\circ} \mathrm{C}$ for $5 \mathrm{~min}$ to get total microbiota, then washed twice in PBS. The final microbial suspension was mixed with an equal volume of $40 \%$ sterile glycerol to a final concentration of $20 \%$, then stored at $-80{ }^{\circ} \mathrm{C}$. In addition, heat-killed microbiota from NS-FMD mice was boiled for $45 \mathrm{~min}$ after washing twice in PBS [19]. For each mouse, $200 \mu$ of microbial suspension $\left(10^{8} \mathrm{CFU} / \mathrm{ml}\right)$ was transplanted to gut microbiota-depleted mice after antibiotic treatment by gastric gavage each day over consecutive 7 days. The dilution coating method was used for determination of microbial concentrations from mice by calculating colonies on solid medium under anaerobic incubation as previously described [29].

\section{Behavioral Test}

On the 5th day after MPTP treatment, the mice began behavioral training once per day for 3 days. Protocols of behavioral tests (pole descent test and traction test) have been described in previous studies $[30,31]$. 
The pole test is a useful method for evaluating bradykinesia in mouse PD models. In the pole descent test, a 0.5-m-long pole, $1 \mathrm{~cm}$ in diameter and wrapped with nonadhesive gauze to facilitate gripping, and with a spherical protuberance, $2 \mathrm{~cm}$ in diameter on top, was placed in the home cage. Mice were placed head-down on the top of the pole and their descent back into the home cage was timed. Timing began when the experimenter released the animal and ended when one hindlimb reached the home cage base. The test was performed three times for each animal, the beginning at the day after the last treatment, and the average of the three trials was calculated for statistical analyses.

The traction test measures muscle strength and equilibrium. In the traction test, the forepaws of the mice were placed on a horizontal rope (diameter $5 \mathrm{~mm}$ ) and observed for $10 \mathrm{~s}$, whereas the hind limb placements were scored from 1 to 4 , with the lowest score indicating the most severe deficit. Animals were assigned a score of 4 for gripping the rope with both hind paws, 3 for gripping the rope with one hind paw, 2 for gripping the rope with both front paws, and 1 for gripping the rope with one front paw. The test was performed three times for each animal, the beginning on the day after the last treatment, and the average of the three trials was calculated for statistical analyses.

\section{Sample Collection and Tissue Preparation}

For collecting feces, mice were placed individually in empty autoclaved cages and allowed to defecate freely in the morning after the day of last treatment. Once feces were formed of each mouse, feces were collected immediately in individual sterile EP tubes on ice and then stored at $-80^{\circ} \mathrm{C}$. For obtaining fresh striatum tissue, mice were deeply anesthetized with isoflurane and then received a transcardiac perfusion of ice-cold sterilized saline. The tissues were immediately stored at $-80^{\circ} \mathrm{C}$.

For immunofluorescence, mice were deeply anesthetized with isoflurane and then transcardially perfused with PBS followed by $4 \%$ paraformaldehyde in $0.01 \mathrm{M}$ PBS, pH 7.4. Tissues were postfixed in $4 \%$ paraformaldehyde at $4{ }^{\circ} \mathrm{C}$ overnight, kept in $20 \%$ sucrose at $4{ }^{\circ} \mathrm{C}$ for $24 \mathrm{~h}$, then transferred into $30 \%$ sucrose at $4{ }^{\circ} \mathrm{C}$ for $24 \mathrm{~h}$, and then embedded in optimal cutting temperature compound (O.C.T. Compound, Tissue-Tek, Torrance, CA, USA). Ten micrometer-thick cross sections were cut using a cryostat microtome (CM1950, Leica, Wetzlar, Germany).

\section{Measurement of Neurotransmitters}

High-performance liquid chromatography with a fluorescence detector (Waters 2475, Milford, MA, USA) was used to measure the levels of striatal DA, 5-HT, and their metabolites, including 3, 4-dihydroxyphenylacetic (DOPAC), homovanillic acid (HVA), and 5-hydroxyindoleacetic acid (5-HIAA), following previous methods with moderate modifications [32]. The separation system (Waters 2695) was used with an Atlantis T3 column $(150 \mathrm{~mm} \times 4.6 \mathrm{~mm}$, $51 \mu \mathrm{m}$, Waters). The mobile phases were composed of water, acetonitrile, and 0.01 M PBS ( $\mathrm{pH} 4.0$ ). Briefly, the striatum was homogenized in $0.1 \mathrm{M}$ perchloric acid $(10 \mu \mathrm{l} / \mathrm{mg}$ of striatum tissue) by sonication and the homogenate was centrifuged at $13,000 \times \mathrm{g}, 4{ }^{\circ} \mathrm{C}$ for $10 \mathrm{~min}$. Then, the supernatants were collected and filtered through a $0.22-\mu \mathrm{m}$ filter and $25 \mu \mathrm{l}$ of sample was injected into the column. In parallel, DA, 5-HT and their metabolites, and a hydrochloride standard (SigmaAldrich, St. Louis, MO, USA) solution were prepared freshly by diluting the stock solution in the mobile phase. The linearity ranges of neurotransmitters were determined by serial concentrations of standard solution before detection.

\section{Western Blot Analysis and Enzyme-Linked Immunosorbent Assay}

Total protein was extracted by homogenizing $20 \mathrm{mg}$ tissue in $200 \mu \mathrm{l}$ radio immunoprecipitation assay lysis buffer (Beyotime, Shanghai, China) with $2 \mu$ phenylmethanesulfonyl fluoride (Solarbio, Beijing, China). After centrifugation of the homogenate at $13,000 \times \mathrm{g}, 4{ }^{\circ} \mathrm{C}$ for $5 \mathrm{~min}$, the supernatant was collected and measured with a BCA protein assay kit (Biosharp, Anhui, China). Forty micrograms of total protein was separated by sodium dodecyl sulfate-polyacrylamide electrophoresis-polyacrylamide gel electrophoresis, blotted onto polyvinylidene difluoride membranes (Millipore, Billerica, MA, USA), and then probed with the following antibodies: mouse anti-tyrosine hydroxylase (TH, 1:1000, MAB318, Millipore), rabbit antiBDNF (1:1000, ab108319, Abcam, Cambridge, MA, USA), rabbit anti- $\beta$ tubulin $(1: 1000,10,068-1-A P$, Proteintech, Wuhan, China) and mouse anti-GAPDH (1:8000, 60,001-1-Ig, Proteintech). Goat anti-rabbit IgG (1:1000, BA1054, Boster, Pleasanton, CA, USA) and goat anti-mouse IgG (1:1000, BA1050, Boster), both conjugated to horseradish peroxidase, were used as secondary antibodies. Protein bands were visualized by incubation with BeyoECL Plus (P0018, Beyotime) for $1 \mathrm{~min}$ and imaged by a Gel Image System (Bio-Rad, Hercules, CA, USA). Densitometry was performed by using the ImageJ software (NIH, Bethesda, MA, USA).

TNF- $\alpha$ and IL- $1 \beta$ concentrations of the striatum were detected using a commercial enzyme-linked immunosorbent assay kit (EK0527/EK0394, Boster). The limit of detection is $15.6-1000 \mathrm{pg} / \mathrm{ml}$ and $12.5-800 \mathrm{pg} / \mathrm{ml}$ respectively. All experimental procedures were performed according to the manufacturer's instructions.

\section{Immunofluorescence and Image Analysis}

Briefly, each mouse brain was cut into coronal slices and slices containing the substantia nigra pars compacta were collected. Brain sections were immersed in $0.01 \mathrm{M}$ sodium citrate buffer 
(pH 6.0) for antigen retrieval and washed in PBS. Then, brain sections were incubated in PBS containing $0.3 \% v / v$ Triton $\mathrm{X}$ 100 and $5 \% \mathrm{v} / \mathrm{v}$ goat serum for $1 \mathrm{~h}$ at $37{ }^{\circ} \mathrm{C}$. Primary antibodies - mouse anti-TH (1:1000, MAB318, Millipore), rabbit anti-glial fibrillary acid protein (GFAP, 1:2000; Z033429, Dako, Glostrup, Denmark), and anti-ionized calcium-binding adaptor molecule 1 (Iba-1, 1:1000, 019-19741, Wako, Osaka, Japan)—were incubated $1 \mathrm{~h}$ at $37^{\circ} \mathrm{C}$. For detection of the primary antibodies, appropriate secondary antibodies, coupled to fluorescein isothiocyanate (FITC)-conjugated goat anti-mouse IgG (1:1000, A0568, Beyotime) and CY3conjugated goat anti-rabbit IgG (1:1000, A0516, Beyotime) were used. Samples were covered with mounting medium (P0126, Beyotime) and examined with an epifluorescence microscope (Nikon Eclipse 80i, Nikon, Tokyo, Japan). For each animal, brain slices containing the major portion of the SN from bregma $-2.92 \mathrm{~mm}$ to $-3.52 \mathrm{~mm}, 10$ representative sections were chosen to be doubly stained with $\mathrm{TH}$ and $\mathrm{NeuN}$ in the both sides of SN, four representative sections were chosen to be doubly stained with TH and GFAP/Iba-1 in the SN. Cells positive for TH, NeuN, GFAP, and Iba-1 were counted in the $\mathrm{SN}$ by the ImageJ analyzer $(\mathrm{NIH})$. The abovementioned sections were quantified for each animal, and each group contained 4-6 animals.

\section{Gut Microbiota Profiling}

Total genomic DNA from fecal samples was extracted using a PowerSoil DNA Isolation Kit (MOBIO, Carlsbad, CA, USA) according to the manufacturer's instructions. DNA quality and concentration were monitored by gel electrophoresis and spectrophotometry (Nanodrop 1000, NanoDrop Technologies, Wilmington, DE, USA). The samples for sequencing $16 \mathrm{~S}$ and 18S rRNA gene V4 regions were prepared following the Illumina protocol for $16 \mathrm{~S}$ and $18 \mathrm{~S}$ metagenomic sequencing library preparation. To generate amplicons, the hypervariable V4 regions of 16S [33] and 18S rRNA [34] were amplified using primers (forward primer, 5'-AYTGGGYDTAAAGNG3' and 5'-CCAGCASCYGCGGTAATTCC-3' respectively; reverse primer, 5'-TACNVGGGTATCTAATCC-3' and 5'ACTTTCGTTCTTGATYRA-3' respectively) with a denaturation step at $95{ }^{\circ} \mathrm{C}$ for $3 \mathrm{~min}$, followed by 30 cycles of denaturation at $95{ }^{\circ} \mathrm{C}$ for $30 \mathrm{~s}$, primer annealing at $55^{\circ} \mathrm{C}$ for $30 \mathrm{~s}$, and primer extension at $72{ }^{\circ} \mathrm{C}$ for 30 s. Upon completion of a

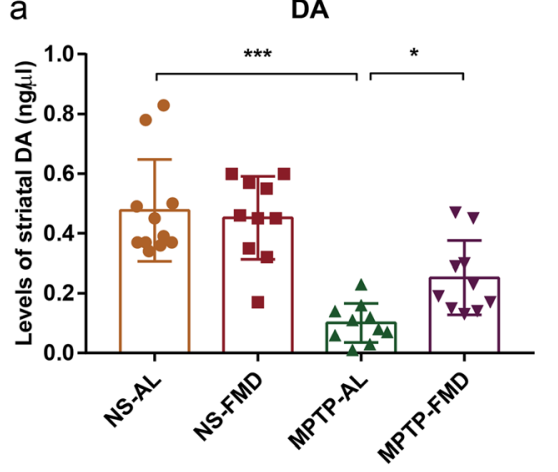

C

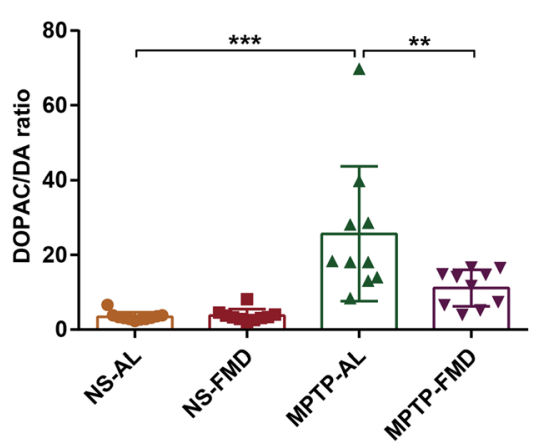

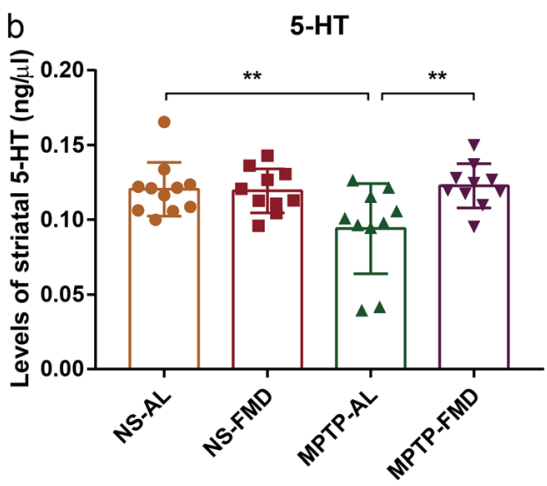

d

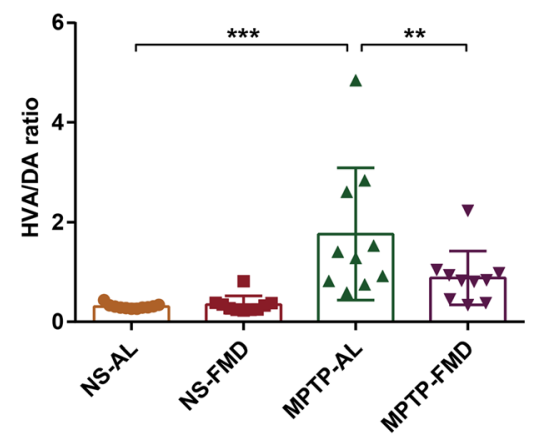

e

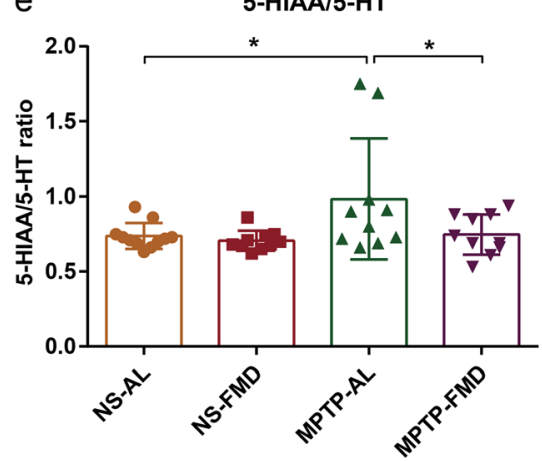

Fig. 2 FMD treatment of PD mice attenuates MPTP-induced reduction of brain neurotransmitters. (a) DA concentration in the mouse striatum, $F_{3,37}=18.462, p<0.001$. (b) 5 -HT concentration in the mouse striatum, $F_{3,37}=4.420, p=0.009$. (c, d) DA turnover in the mouse striatum. The ratio of DOPAC/DA (c) and HVA/DA (d) represents the degree of DA turnover, $F_{3,37}=12.792, p<0.001$ and $F_{3,37}=9.221, p<0.001$, respectively. (e) 5-HT turnover in the mouse striatum. The ratio of 5HIAA/5-HT represents the degree of 5-HT turnover, $F_{3,37}=3.497, p=$ 0.025 . Statistical comparison by one-way ANOVA with post hoc comparisons of LSD; data represent the means $\pm \mathrm{SEM} ; * p<0.05$, $* * p<0.01$, $* * * p<0.001$. NS-AL group $(n=11), n=10$ mice in the other three groups 
cycling, a final extension at $72{ }^{\circ} \mathrm{C}$ for $5 \mathrm{~min}$ was done and then the reaction was held at $4{ }^{\circ} \mathrm{C}$. The PCR products were then purified with magnetic beads (Agencourt AMPure XP, Beckman, CA, USA), and then a second round of PCR amplification was implemented to introduce dual indices and sequencing adapters. The purified products were quantified using a fluorometric quantification method that uses dsDNA binding dyes (Qubit ${ }^{\circledR}$ dsDNA HS Assay Kits, Life Technologies, Carlsbad, CA, USA) and pooled into a library after normalization. RNA sequencing was performed on an Illumina HiSeq 2500 to generate pair-end 250 bp reads.

The data analysis was performed by the QIIME v1.9.1 platform [35]. Raw sequencing data was filtered using FASTQC according to the phred scores, and the reads (110149.1 \pm 2708.5 reads per sample for $16 \mathrm{~S}$ rRNA analysis and $137167.3 \pm 5176.9$ reads per sample for $18 \mathrm{~S}$ rRNA analysis) were trimmed if the average phred score in the window ( $5 \mathrm{bp}$ in size, 1 bp step length) was less than 20 . Reads containing ambiguous " $\mathrm{N}$ " or with length $<150 \mathrm{bp}$ were discarded. Paired reads were merged into a tag sequence according to their overlap. By aligning with Sliva, Unite, and RDP database, the chimeras were removed using the algorithm of usearch61. High-quality sequences without chimeras were clustered into OTUs (operational taxonomic units), with a similarity of $97 \%$ [36]. OTU picking was performed using Uclust on the software platform QIIME v1.9.1. Nonrepetitive ones were extracted from optimized sequences to reduce the redundant computation. Sequences without repeats were removed. OTUs with 97\% similarity were clustered. Chimeras were removed during the clustering process and representative sequences of OTU were obtained. All of the optimized sequences were mapped to the representative OTU and those with were selected to generate OTU table. OTUs with low abundance (the threshold value was 0.00001 ) were screened and filtered. The longest sequence of each OTU was selected as a representative sequence which was annotated by comparing to the Greengenes database (Release 13.5, http://greengenes.secondgenome. com/) by the RDP-classifier method [37, 38].

Alpha diversity and beta diversity analyses were performed based on rarefied OTUs. For 16S rRNA analysis, the OTU table was rarified to 72,130 reads, whereas for $18 \mathrm{~S}$ rRNA analysis, the OTU table was rarified to 82,869 reads. The rarefaction analysis based on mothur v.1.21.1 was conducted to reveal the diversity indices, including Chaol (http://www. mothur.org/wiki/Chao) and Shannon diversity indices (http:// www.mothur.org/wiki/Shannon). Beta diversity was used to evaluate differences of samples in species diversity and was characterized by principal coordinate analysis ( $\mathrm{PCoA}$ ) based on Bray-Curtis distance. The Vegan 2.0 package was used to generate a PCoA figure and the differences among the groups were tested by ANOSIM (analysis of similarity). The software package of ggplot2 and pheatmap provided by $\mathrm{R}$ language were used to generate histogram and heatmap separately to
Fig. 3 FMD treatment of PD mice prevents the MPTP-induced loss in brain dopaminergic neurons and reduction of TH expression. (a) Representative immunofluorescence staining for $\mathrm{TH}$ and $\mathrm{NeuN}$ in the right and left SN respectively. Scale bar is $100 \mu \mathrm{m}$. (b) Quantitative analysis of the number of TH-positive cells in the left $\mathrm{SN}, F_{3,12}=$ $34.100, p<0.001, n=4$ mice per group. (c) Quantitative analysis of the number of TH-positive cells in the right $\mathrm{SN}, F_{3,12}=51.657, p<0.001$, $n=4$ mice per group. (d) Quantitative analysis of the number of NeuNpositive cells in the left SN, $F_{3,12}=32.374, p<0.001, n=4$ mice per group. (e) Quantitative analysis of the number of NeuN-positive cells in the right SN, $F_{3,12}=38.919, p<0.001, n=4$ mice per group. (f) Representative western blot of striatal TH expression. (g) Band intensity was quantified with the ImageJ software and quantitative data for $\mathrm{TH}$ following normalization to GAPDH, $F_{3,24}=16.732, p<0.001, n=7$ mice per group. Statistical comparison by one-way ANOVA with post hoc comparisons of LSD; data represent the means \pm SEM; $* * p<0.01$, $* * * p<0.001$

describe the composition and diversity of the communities with OTU table as an input file.

\section{Fecal Short-Chain Fatty Acids Profiling}

Fecal SCFA concentrations were determined using gas chromatography-mass spectrometry. Fecal samples collected before euthanasia were stored at $-80^{\circ} \mathrm{C}$ and then thawed to room temperature for processing. Briefly, $50 \mathrm{mg}$ feces were ultrasonicated with $100 \mu \mathrm{l}$ of water in a bath ultrasonicator for $5 \mathrm{~min}$ and vortex-mixed for $2 \mathrm{~min}$ and then centrifuged at $4{ }^{\circ} \mathrm{C}$ at $16,000 \times g$ for $15 \mathrm{~min}$. An aliquot $(100 \mu \mathrm{l})$ of the supernatant was acidified by adding $50 \mu \mathrm{l} 50 \%(v / v)$ sulfuric acid. After vortexing and standing for $2 \mathrm{~min}$, the organic acids were extracted by adding $225 \mu \mathrm{l}$ of $\mathrm{n}$-hexane [39, 40], and supernatants were measured by gas chromatography-mass spectrometry on a Trace 1300/Exactive GC apparatus (Thermo Fisher Scientific, Waltham, MA, USA) equipped with flame electron impact ionization and an Rtx-WAX column $(30 \mathrm{~m} \times$ $0.25 \mathrm{~mm} \times 0.25 \mu \mathrm{m}$, Bellefonte, PA, USA).

\section{Fecal Untargeted Metabolomics}

Fecal untargeted metabolomics was based on liquid chromatography-mass spectrometry. Fecal samples collected before euthanization were stored at $-80^{\circ} \mathrm{C}$ and then thawed to room temperature for processing. Water, methanol, and acetonitrile, in order, were selected as solvent for extraction. In brief, each fecal sample of $80 \mathrm{mg}$ was extracted 3 times with $200 \mu \mathrm{l}$ of solvent by ultrasonication in a noncontact ultrasonic cell grinder (Scientz08-I, Zhejiang, China) for $15 \mathrm{~min}$, vortex-mixing for $2 \mathrm{~min}$, and then centrifugation at $4{ }^{\circ} \mathrm{C}$ at $16,000 \times g$ for $15 \mathrm{~min}$. The supernatants from the three extractions were transferred to a new $1.5 \mathrm{ml}$ centrifuge tube and vortex-mixed [41]. The mixed extracts were dried with CentriVap (Labconco, Kansas City, USA), redissolved with $200 \mu \mathrm{l}$ of mixed solvent (water/methanol/acetonitrile = $1: 1: 1$, and then centrifuged at $4{ }^{\circ} \mathrm{C}$ at $16,000 \times g$ for $5 \mathrm{~min}$. 
a

NS-AL

NS-FMD
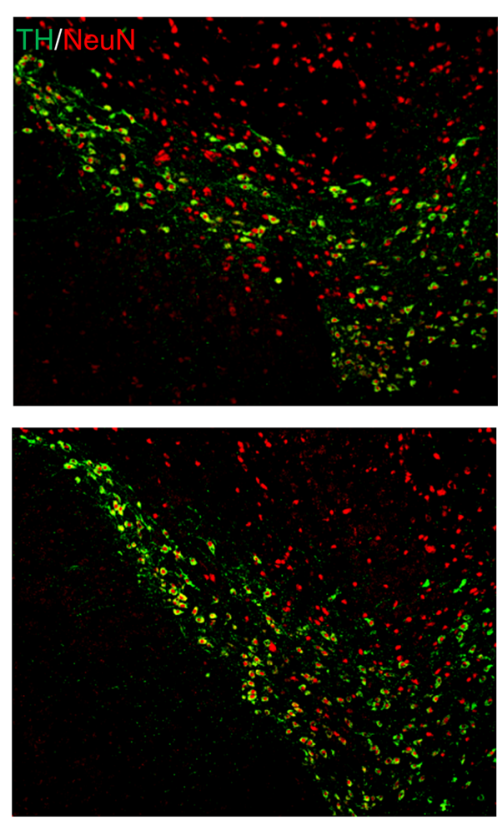

MPTP-AL

MPTP-FMD
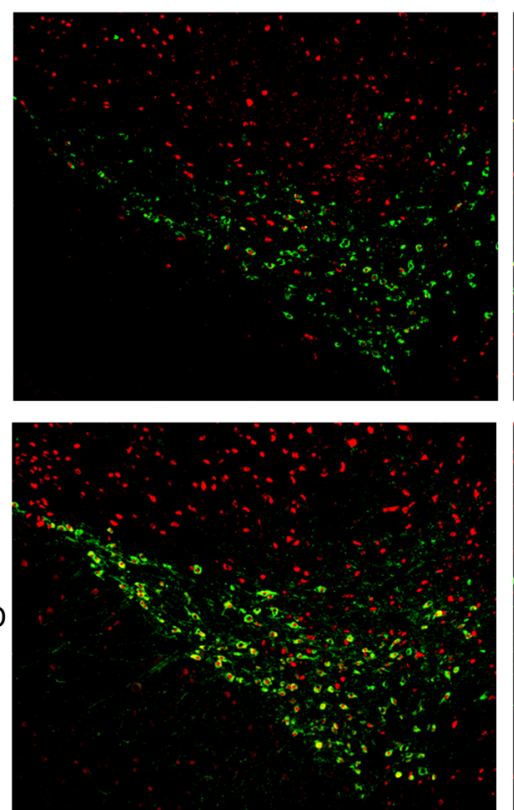

Right
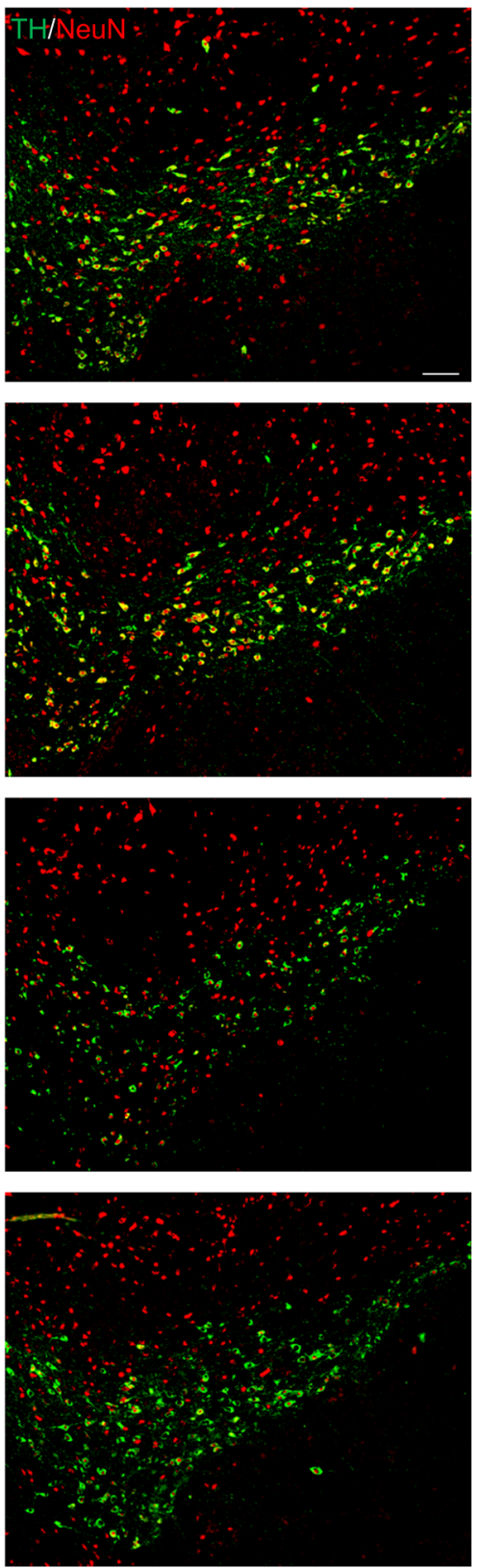

b 2

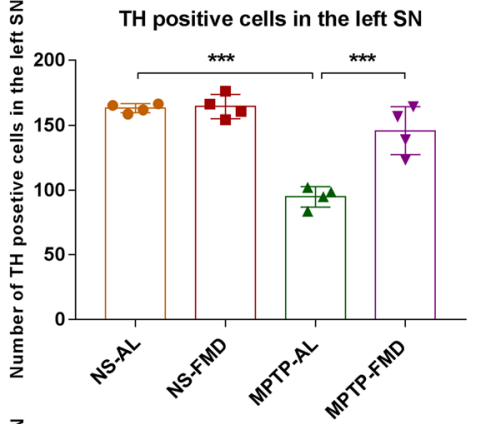

C 2

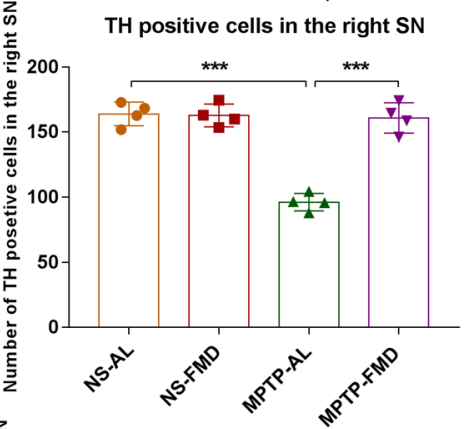

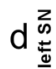

NeuN positive cells in the left $\mathrm{SN}$

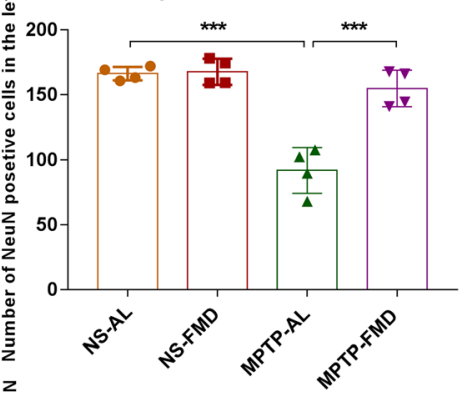

$\mathrm{e} \stackrel{2}{\frac{2}{5}} \quad$ NeuN positive cells in the right $\mathrm{SN}$

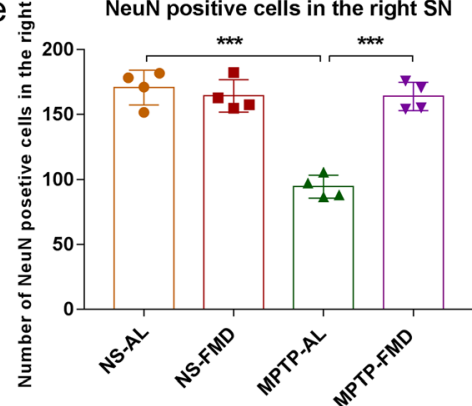

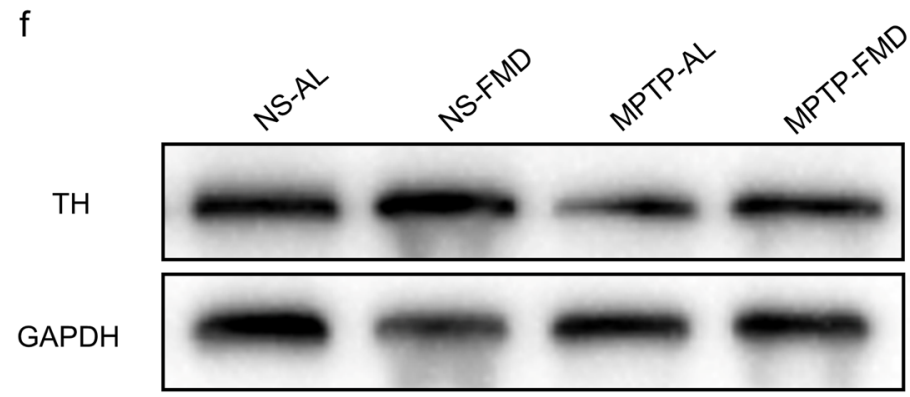

$62 \mathrm{KD}$

$36 K D$

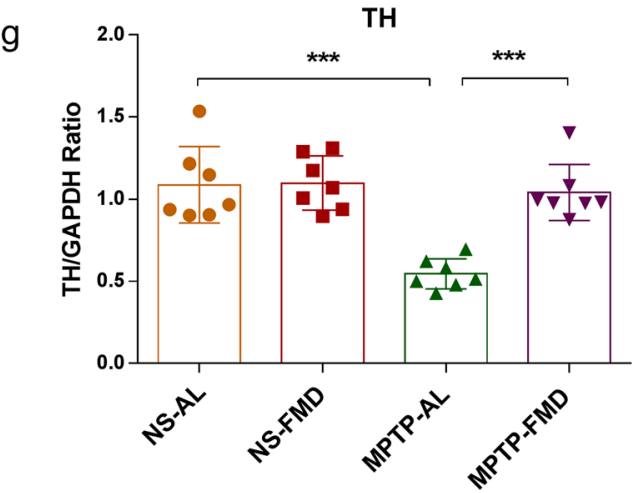



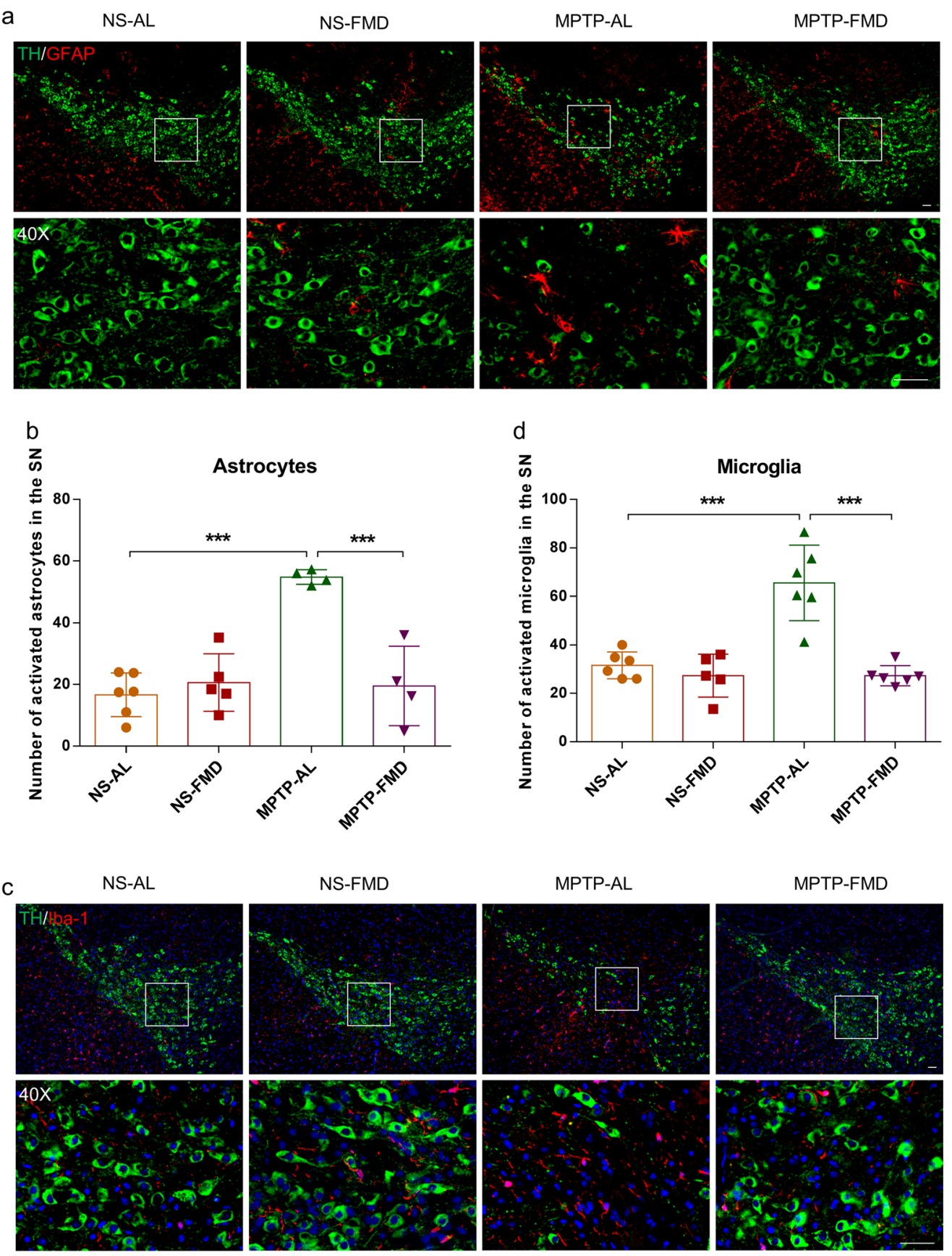

e
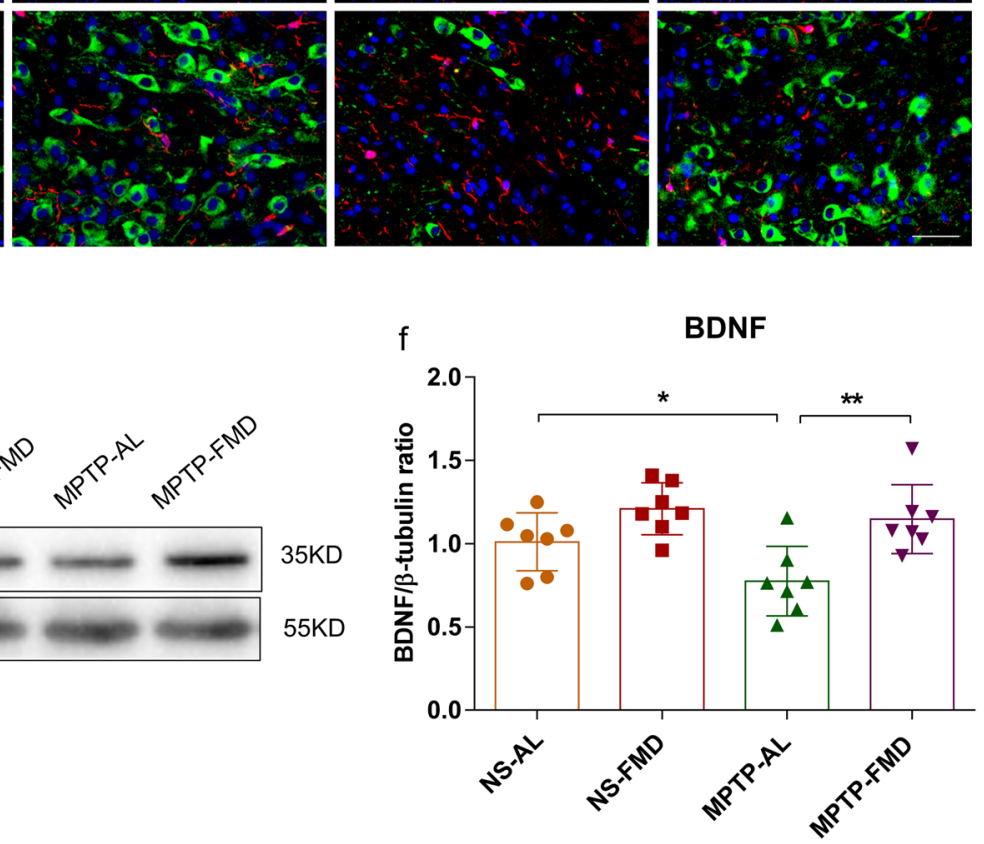
Fig. 4 FMD alleviates MPTP-induced neuro-inflammation by retaining BDNF levels. (a) Double immunofluorescence staining for TH (green) and GFAP (red) in the SN. Scale bar is $100 \mu \mathrm{m}$. (b) Quantitative analysis of the number of GFAP positive cells in each group, $F_{3,15}=18.574$, $p<0.001$. NS-AL group $(n=6)$, NS-FMD group $(n=5)$, MPTP-AL group $(n=4)$, and MPTP-FMD group $(n=4)$. (c) Double immunofluorescence staining of TH (green) and Iba-1 (red) in the SN. Scale bar is $100 \mu \mathrm{m}$. (d) Quantitative analysis of the number of microglia in each group, $F_{3,19}=21.832, p<0.001$. NS-FMD group $(n=5), n=6$ mice in the other three groups. (e) Representative western blot of striatal BDNF expression. (f) Band intensity was quantified with the ImageJ software and quantitative data for $\mathrm{TH}$ following normalization to GAPDH, $F_{3,24}=$ 7.375, $p=0.001, n=7$ mice per group. (g) ELISA for striatal TNF- $\alpha$ expression, $F_{3,28}=6.316, p=0.002, n=8$ mice per group. (h) ELISA for striatal IL-1 $\beta$ expression, $F_{3,20}=13.529, p<0.001, n=6$ mice per group. Statistical comparison by one-way ANOVA with post hoc comparisons of LSD; data represent the means $\pm \mathrm{SEM} ;{ }^{*} p<0.05,{ }^{* *} p<0.01$, $* * * p<0.001$

The supernatants, containing fecal metabolomics, were measured by liquid chromatography-mass spectrometry Q Exactive Plus (Thermo Fisher Scientific) equipped with a

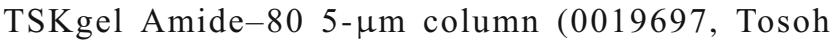
Corporation, Tokyo, Japan). For normalization, the spectral counts of each metabolite were divided by the total spectral counts of all metabolites from the same sample. The data transformation is log transformation. The tool used for statistical analysis is Compound Discoverer 2.1. The database used for identification of peaks is mzCloud.

\section{Statistical Analysis}

Microbiome population and untargeted metabolomics statistics are described in detail above. Excluding these, the SPSS 22.0 software was used for data analysis. Statistical analysis was conducted by one-way ANOVA with an LSD post hoc assay. Data are presented as mean \pm SEM (standard error of the mean), and $p<0.05$ was set as the threshold for significance $(* p<0.05, * * p<0.01, * * * p<0.001)$. All data were displayed using GraphPad Prism version 7 (GraphPad, Inc., La Jolla, CA, USA).

\section{Results}

\section{FMD Cycling Prevents Motor Dysfunction of PD Mice Without an Overall Reduction in Calorie Intake}

We examined the effects of FMD, fasting 3 days out of every 7 days for 3 cycles ( 3 days of FMD followed by 4 days of refeeding, every 7 days per cycle), in a murine model of MPTP-induced PD (Fig. 1a). On the day after the last day of 3-day FMD period in per cycle, FMD-treated mice cotreated with NS (NS-FMD) lost $21.8 \%$ body weight and cotreated with MPTP (MPTP-FMD) caused a $22.3 \%$ loss of body weight (Fig. 1b). During each FMD cycle, the mice regained most of the weight upon refeeding and the body weight of FMD mice were almost equal to AL mice on the first day after per cycle (Fig. 1b). Although FMD mice (NS-FMD and MPTP-FMD) were severely calorically restricted during the 3-day FMD, they compensated for this restriction by overeating during the 4-day refeeding period, resulting in average calorie intake per day in 7-day cycle 1 and cycle 3 equivalent for the FMD and the AL groups, whether combined with NS or MPTP (Fig. 1c). Food intake of MPTP-AL mice in cycle 2 was less than that in the other three groups (Fig. 1c).

To assess the potential neuroprotective effects of FMD on motor function in MPTP-induced PD mice, we subjected mice to a pole test to measure the total descent time for evaluation of bradykinesia (Fig. 1d) and a traction test to measure the traction score for evaluation of muscle strength and equilibrium (Fig. 1e). Compared with NS-AL mice, MPTP-AL mice exhibited significant locomotor deficits, including $64.3 \%$ longer in pole descent time ( $p<0.001 v s$ NS-AL mice) and $33.7 \%$ lower in traction test scores $(p<0.001$ vs NS-AL mice). However, MPTP-FMD mice had significantly better motor performance both in the pole test, which showed as decreased by $20.4 \%$ in pole descent time $(p<0.001$ vs MPTP-AL mice) and traction test, which showed as increased by $33.8 \%$ in the traction test scores $(p<0.001$ vs MPTP-AL mice). Intriguingly, there was no obvious difference in motor performance between NS-AL mice and NS-FMD mice ( $p=$ 0.518 ), suggesting that FMD treatment has no effect on normal mice (Fig. 1d, e). Therefore, FMD treatment appears to favor prevention of motor dysfunction in $\mathrm{PD}$ mice.

\section{FMD Rescues Striatal Dopamine and Serotonin Levels in PD Mice}

To evaluate potential neuroprotective effects of FMD on brain function, striatal neurotransmitters, DA and 5-HT, and their metabolites (DOPAC, HVA, 5-HIAA), were measured by fluorescence detection following high-performance liquid chromatography. As expected, striatal DA levels were dramatically decreased by $78.8 \%$ in MPTP-AL mice (treated with MPTP and ad libitum) compared with NS-AL mice (treated with normal saline and ad libitum $)(p<0.001 v s$ NS-AL mice), but increased by $149.5 \%$ in MPTP-FMD (treated with MPTP and fasting mimicking diet) mice compared with MPTP-AL mice ( $p<0.05 v s$ MPTP-AL mice) (Fig. 2a). Similarly, 5-HT was also decreased in MPTP-AL mice compared with NS-AL mice ( $p<0.01 v s$ NS-AL mice), and increased in MPTP-FMD ( $p<0.01$ vs MPTP-AL mice) (Fig. 2b). No significant differences for DA and 5-HT were observed between NS-FMD mice (treated with normal saline and fasting mimicking diet) and NS-AL mice (Fig. 2a, b), suggesting FMD has no effect of DA and 5-HT on normal mice. Intriguingly, DOPAC/DA and HVA/DA ratios in the striatum increased by $640.5 \%$ and $469.7 \%$ in MPTP-AL mice, 

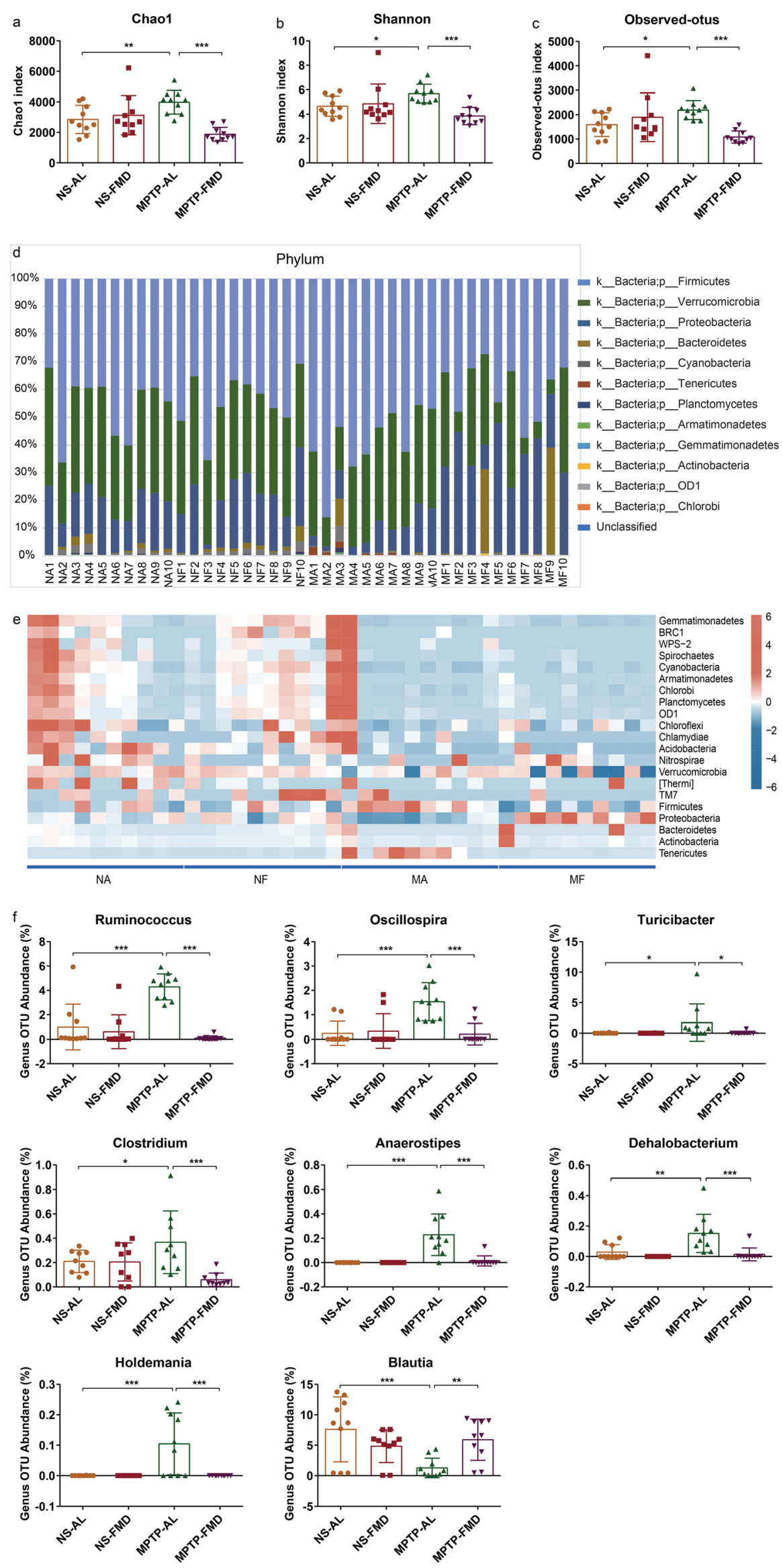
Fig. 5 FMD modifies the dysbiosis of gut microbiota induced by MPTP based on 16S rRNA sequencing. (a-c) Analysis of alpha diversity-based, $16 \mathrm{~S}$ rRNA sequencing-predicted gut microbiota richness by the Chao 1 index (a), diversity by the Shannon index (b) and the number of OTUs by observed_otus (c). (d) Comparison of the fecal microbiota structures and distribution at the phylum-based 16S rRNA sequencing level. (e) Heatmap based on the Bray-Curtis distance analysis of $16 \mathrm{~S}$ rRNA showing relative abundance of gut microbiota at the phylum level. (f) Relative abundance of gut microbiota changed significantly at the genus level, based on $16 \mathrm{~S}$ rRNA sequencing. NA $=$ NS-AL group; NF $=$ NS-FMD group; $\mathrm{MA}=\mathrm{MPTP}-\mathrm{AL}$ group; $\mathrm{MF}=\mathrm{MPTP}-\mathrm{FMD}$ group. $n=10$ mice per group

respectively $(p<0.001$ and $p<0.001 v s$ NS-AL mice, respectively), suggesting increased DA turnover. In response to FMD treatment, these two ratios were decreased by $56.5 \%$ and $49.9 \%$ in MPTP-FMD mice compared with MPTP-AL mice $(p<0.01$ and $p<0.01 v s$ MPTP-AL mice, respectively), which can be interpreted as a reduction in dopaminergic turnover rate (Fig. 2c, d). Similar to DA turnover ratio, the turnover rate of 5-HT, as judged by the 5-HIAA/5-HT ratio, was also increased by $33.3 \%$ in MPTP-AL mice compared with NS-AL mice ( $p<0.05 v s$ NS-AL mice), but was $24.0 \%$ lower in MPTP-FMD mice compared with MPTP-AL mice $(p<0.05$ vs MPTP-AL mice), indicating FMD reduced the MPTP-induced alterations in 5-HT turnover (Fig. 2e). These results suggest that FMD participates in the metabolism of brain neurotransmitters by suppressing the MPTP-induced decline of striatal DA and 5-HT and increase of their turnover.

\section{FMD Blocks the MPTP-Induced Decline in Dopaminergic Neurons and TH Levels in PD Mice}

To examine the effects of FMD on survival of dopaminergic neurons in the $\mathrm{SN}$ and to verify whether TH expression corresponded with DA levels, we characterized TH expression by western blot analysis in the striatum and immunofluorescence staining in the SN. Immunofluorescence staining in the $\mathrm{SN}$ revealed a significant loss $(41.8 \%$ in the left $\mathrm{SN}, 41.3 \%$ in the right $\mathrm{SN}$ ) of $\mathrm{TH}^{+}$cells in MPTP-AL mice (treated with MPTP and ad libitum) compared with NS-AL mice (treated with normal saline and ad libitum) (both $p<0.001 v s$ NS-AL mice), whereas MPTP-FMD mice (treated with MPTP and fasting mimicking diet) had $53.6 \%$ and $67.1 \%$ more $\mathrm{TH}^{+}$cells than MPTP-AL mice in the left and right $\mathrm{SN}$ respectively (both $p<0.001 v s$ MPTP-AL mice), and there was no significant difference between NS-AL mice and NS-FMD mice (treated with normal saline and fasting mimicking diet) (Fig. 3a-c). To verify that this was not simply phenotypic regulation of $\mathrm{TH}$, neurons were semi-quantified by NeuN staining. MPTP-AL mice had $44.7 \%$ (in the left SN) and $44.6 \%$ (in the right $\mathrm{SN}$ ) less $\mathrm{NeuN}^{+}$cells than NS-AL mice (both $p<0.001$ vs NS-AL mice), and FMD rescued the loss with $68.3 \%$ and $73.3 \%$ in the left and right SN compared with MPTP-AL mice (both $p<0.001$ vs MPTP-AL mice) (Fig.3a- e). These counts confirmed the loss of dopaminergic neurons in the SN induced by MPTP and rescued by FMD. Western blot analysis of striatal tissue showed that TH expression in MPTP-AL mice was reduced by $43.8 \%$ compared with NSAL mice ( $p<0.001 v s$ NS-AL mice), and FMD inhibited the reduction of TH expression by $52.0 \%$ ( $p<0.001 v s$ MPTP-AL mice) (Fig. 3f, g). These results demonstrate that the neuroprotective effects of FMD involve the suppression of dopaminergic neuron loss in the $\mathrm{SN}$ and the decreased striatal $\mathrm{TH}$ expression in MPTP-induced PD mice, but not in normal mice.

\section{FMD Retains the Balance of Brain Glia and BDNF in PD Mice}

Glial cells and neurotrophins play important roles in maintaining homeostasis of central nervous system. Disruption of their function results in lots of central nervous system diseases, including PD. The neurodegeneration of dopaminergic neurons could be prevented by stimulation of the production and release of BDNF via inhibition of inflammation [42].

To explore whether brain glia population increase and are accompanied by abnormal decreases in BDNF, we performed immunofluorescence staining to characterize the population of microglia and astrocytes in the SN. Double immunofluorescence staining for $\mathrm{TH}$ (dopaminergic neuron marker) and GFAP (astrocyte marker) revealed the presence of a higher number of astrocytes in the SN by $229.0 \%$ in MPTP-AL mice (treated with MPTP and ad libitum) than NS-AL mice (treated with normal saline and ad libitum) $(p<0.001$ vs NS-AL mice), but FMD significantly decreased the number of astrocytes around dopaminergic neurons by $64.4 \%$ in MPTP-FMD mice (treated with MPTP and fasting mimicking diet) $(p<0.001 v s$ MPTP-AL mice) (Fig. 4a, b). Similarly, coexpression of TH with Iba-1 (microglia marker) showed that microglia population in the $\mathrm{SN}$ increased by over 2 -fold in MPTP-AL mice compared with NS-AL mice $(p<0.001 v s$ MPTP-AL mice), and in MPTP-FMD mice, microglia population decreased by $58.4 \%$ compared with MPTP-AL mice $(p<0.001 v s$ MPTP-AL mice) (Fig. 4c, d). Detection of BDNF levels in the striatum by western blot analysis showed that MPTP-AL mice exhibited lower BDNF levels relative to NS-AL mice $(p<0.05 v s$ NS-AL mice) and higher levels in MPTP-FMD mice compared with MPTP-AL mice $(p<0.01$ $v s$ MPTP-AL mice) (Fig. 4e, f). Correspondingly, NS-AL mice were indistinguishable from NS-FMD mice (treated with normal saline and fasting mimicking diet) in regard to population of brain glial cells and BDNF levels.

To further verify whether the imbalance of glial cells and BDNF coupled by inflammation, we examined the levels of TNF- $\alpha$ and IL- $1 \beta$ in the striatum by ELISA. MPTP resulted in increases by $40.9 \%$ and $46.0 \%$ in TNF- $\alpha$ and IL- $1 \beta$ levels respectively, in MPTP-AL mice $(p<0.01$ and $p<0.001 v s$ 

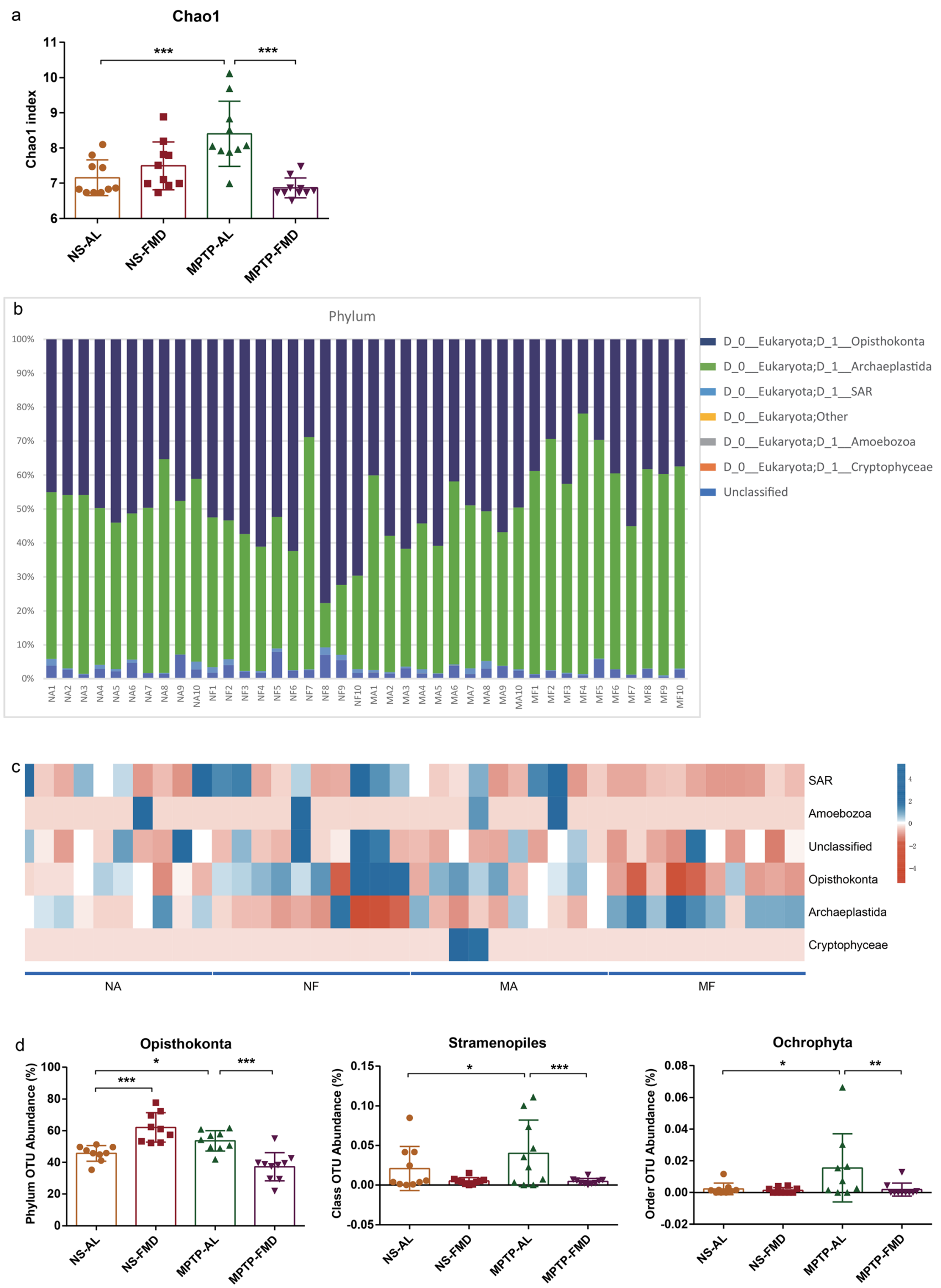
Fig. 6 FMD modifies the dysbiosis of gut microbiota induced by MPTP based on 18S rRNA sequencing. (a) Analysis of alpha diversity based on $18 \mathrm{~S}$ rRNA sequencing-predicted gut microbiota richness by the Chao 1 index. (b) Comparison of fecal microbiota structures and distribution at the phylum level, based on 18S rRNA sequencing. (c) Heatmap based on the Bray-Curtis distance analysis of $18 \mathrm{~S}$ rRNA showing relative abundance of gut microbiota at the phylum level. (d) Relative abundance of gut microbiota changed significantly based on 18S rRNA sequencing. $\mathrm{NA}=\mathrm{NS}-\mathrm{AL}$ group; NF = NS-FMD group; MA = MPTP-AL group; $\mathrm{MF}=\mathrm{MPTP}-\mathrm{FMD}$ group. $n=10$ mice per group

NS-AL mice), but FMD treatment restored TNF- $\alpha$ and IL-1 $\beta$ levels by $40.3 \%$ and $30.7 \%$ in MPTP-FMD mice compared with MPTP-AL mice $(p<0.001$ and $p<0.001 v s$ MPTP-AL mice) (Fig. 4g, h). Taken together, these data indicate that FMD treatment probably retain the balance of brain glia and BDNF in PD mice via inhibition of neuro-inflammation.

\section{FMD Modifies the Dysbiosis of Gut Microbiota Induced by MPTP}

To test the possibility that FMD induce the alleviation of PD by altering gut microbiota composition, fecal microbiota communities were profiled by $16 \mathrm{~S}$ rRNA and $18 \mathrm{~S}$ rRNA gene amplicon sequencing.

Based on 16S rRNA gene amplicon sequencing for identifying bacteria, FMD caused clear alterations in the richness of gut microbiota species (i.e., Chaol index), microbiota diversity (i.e., Shannon index), and number of operational taxonomic units (OTU) (i.e., observed_otus) in PD mice, as indicated by the alpha diversity. Across all these indices, MPTP-AL mice (treated with MPTP and ad libitum) were significantly higher than both NS-AL mice (treated with normal saline and ad libitum) and MPTP-FMD mice (treated with MPTP and fasting mimicking diet), indicating that PD mice had greater microbiota diversity (Fig. 5a-c). Moreover, significant alterations at the phylum level were observed in the microbiota communities between MPTP-AL mice and NS-AL mice, shown as higher OTU abundances of Firmicutes and Tenericutes and lower OTU abundance of Proteobacteria in MPTP-AL mice $(p<0.01, p<0.001$, and $p<0.05 v s$ NS-AL mice, respectively), and FMD inhibited the alteration of these bacteria (Supplementary Fig. 1a), suggesting gut microbial dysbiosis in PD mice, and FMD treatment prevented gut microbial dysbiosis in PD mice (Fig. 5d). The heatmap revealed significant differences in relative abundance across the four groups at the phylum level (Fig. 5e). More specifically, at the genus level, higher OTU abundances were observed for Ruminococcus, Oscillospira, Turicibacter, Clostridium, Anaerostipes, Dehalobacterium, and Holdemania in MPTPAL mice compared with NS-AL mice and MPTP-FMD mice. On the contrary, genus OTU abundance for Blautia was decreased in MPTP-AL mice compared with NS-AL mice and was strikingly increased in MPTP-FMD mice relative to
MPTP-AL mice (Fig. 5f). Not only at the genus level, significant differences of microbiota communities at the phylum, class, order, and family levels were also observed (Supplementary Fig. 1a-d). Differences in bacterial community structures are apparent in principal coordinate analyses (PCoA) of beta diversity Bray-Curtis distance, which revealed a distinct clustering of microbiota composition between the NS-AL, NS-FMD, MPTP-AL, and MPTP-FMD groups $(R=$ $0.2702, p=0.001$ ) (Supplementary Fig. 2a).

Based on 18S rRNA gene amplicon sequencing for identifying fungi, the alpha diversity index of Chao1, representative of microbial diversity in MPTP-AL mice, was higher than NSAL mice. However, the alpha diversity of MPTP-FMD mice was similar to that of NS-AL mice, indicating that FMD was able to prevent the MPTP-induced increase in alpha diversity (Fig. 6a). The overall structure of the gut microbiota alteration at the phylum level was observed (Fig. 6b). Correspondingly, relative abundance at the phylum level was different under different treatments (Fig. 6c). Specifically, the relative abundances of Opisthokonta at the phylum level, Stramenopiles at the class level, and Ochrophyta at the order level in MPTP-AL mice were higher than NS-AL mice and decreased in MPTPFMD mice (Fig. 6d), indicating FMD prevents MPTPinduced alterations in gut microbiota. Differences in bacterial community structures are apparent in PCoA of beta diversity Bray-Curtis distance $(R=0.4302, p=0.001)$, which revealed a distinct clustering of microbiota composition between the NS-AL, NS-FMD, MPTP-AL, and MPTP-FMD groups (Supplementary Fig. 2b). According to these data, PD mice display gut microbial dysbiosis, and FMD can modulate the microbiota composition to achieve beneficial effects on PD mice induced by MPTP.

\section{Gut Microbiota Contributes to FMD-Induced Neuroprotection for PD}

To investigate whether the FMD-induced microbial shift directly contributes to neuroprotection in PD mice, microbiota from NS-AL (treated with normal saline and ad libitum) and NS-FMD mice (treated with normal saline and fasting mimicking diet), referred as AL microbiota and FMD microbiota, respectively, were transplanted to antibiotic-pretreated PD mice, denoted the PD-NA group (treated with AL microbiota) and PD-NF group (treated with FMD microbiota), respectively (Fig. 7a). The control subjects, treated with antibiotic cocktails and MPTP firstly, and then treated with $20 \%$ glycerol in sterile PBS by gastric gavage, were denoted the PD-PBS/G group. Striatal DA concentration of PD-NF mice was strikingly higher than both PD-PBS/G and PD-NA mice. Similarly, 5HT concentration increased significantly in PD-NA mice compared with $\mathrm{PD}-\mathrm{PBS} / \mathrm{G}$ mice and was also higher in PDNF mice than PD-PBS/G and PD-NA mice. Furthermore, microbiota from NS-FMD mice were first heat-killed, then 


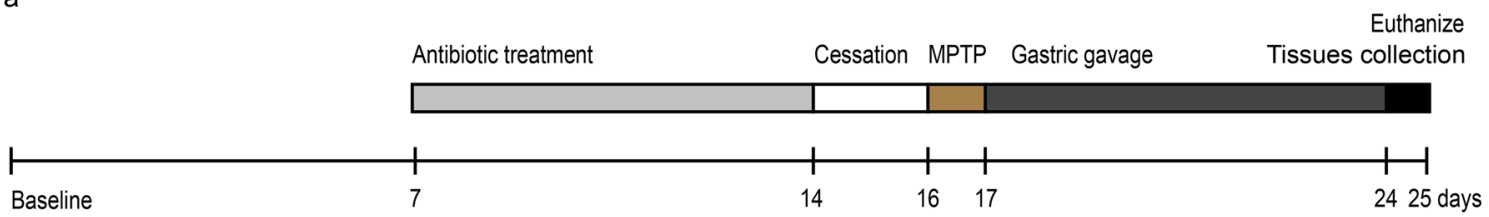

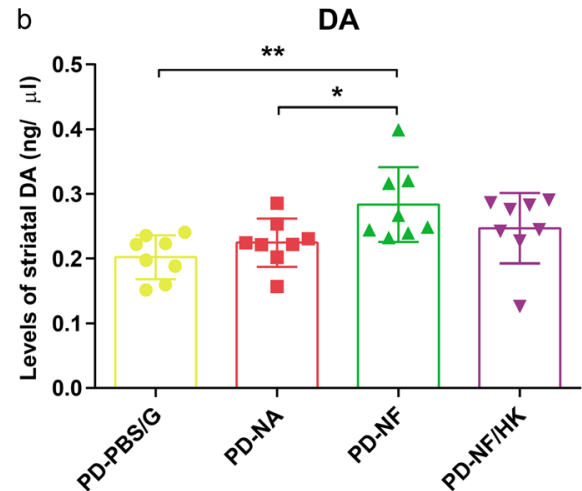

Fig. 7 Gut microbiota contribute to FMD-induced neuroprotection in PD. (a) Experimental scheme of antibiotic cocktail treatment and fecal microbiota transplantation. (b, c) DA (b) and 5-HT (c) concentrations in the striatum of mice with transplanting different microbiota following

transplanted to antibiotic-pretreated PD mice, denoted the PD$\mathrm{NF} / \mathrm{HK}$ group. 5-HT concentration decreased significantly in PD-NF/HK mice compared with PD-NF mice. Similarly, DA concentration of PD-NF/HK mice tended to be lower than that in PD-NF mice, but there was no significance (Fig. 7b, c). Therefore, these findings support the hypothesis that the neuroprotective effects of FMD on PD mice are mediated by the gut microbiota and microbiota need to be metabolically active.

\section{Short-Chain Fatty Acids Concentrations of Feces Influenced by Gut Microbiota Alteration}

SCFAs, ranging from two to five carbon atom chains, including acetic acid, propionic acid, butyric acid, isobutyric acid, valeric acid, and isovaleric acid, are products of gut microbial metabolism, with the amount of each dependent on the microbiome (composition of bacterial species in the gut) [43]. Therefore, we detected the fecal SCFA concentrations in the different treatment groups by gas chromatography-mass spectrometry. Interestingly, propionic acid and isobutyric acid concentrations significantly decreased in MPTP-AL mice (treated with MPTP and ad libitum) compared with NS-AL mice (treated with normal saline and ad libitum), whereas a sharp increase was noted in MPTP-FMD mice (treated with MPTP and fasting mimicking diet) relative to MPTP-AL mice (Fig. 8a, b). Inversely, butyric acid and valeric acid concentrations displayed striking increases in MPTP-AL mice when compared with NS-AL mice and decreased in MPTP-FMD mice when compared with MPTP-AL mice (Fig. 8c, d). In agreement with the alterations in gut microbiota, there were

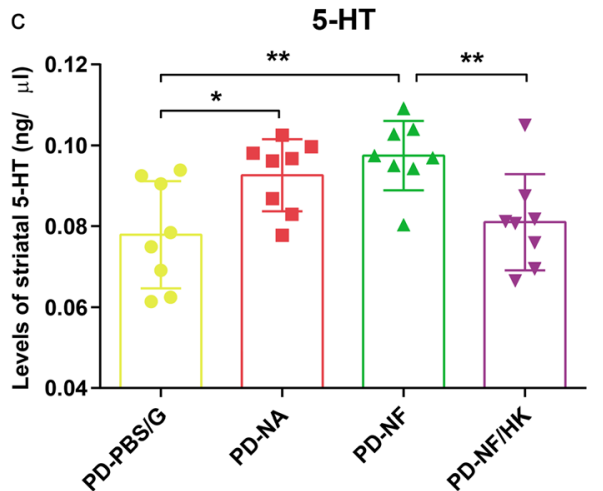

pretreatment with antibiotic cocktails, $F_{3,28}=4.356, p<0.001$ and $F_{3,28}=5.917, p=0.003$, respectively, $n=8$ mice per group. Statistical comparison by one-way ANOVA with post hoc comparisons of LSD; data represent the means $\pm \mathrm{SEM} ; * p<0.05, * * p<0.01, * * * p<0.001$

no differences in SCFAs between NS-AL mice and NS-FMD mice (treated with normal saline and fasting mimicking diet).

\section{FMD Affects Metabolite Spectrum of Mice}

To further determine the potential mechanism of FMDinduced neuroprotection, liquid chromatography-mass spectrometry-based untargeted metabolomics was carried out on feces obtained from the four groups of mice. Based on untargeted fecal metabolomics profiles, a total of 1786 metabolites were detected. Of those, 670 were identified metabolites, whereas 1116 lacked full structural identification (unknown metabolites). Forty-five significantly changed metabolites are listed (Table 1). To characterize the global metabolic differences among groups, a principal component analysis was applied. Fecal metabolite profiles associated with the AL group were tightly clustered, whereas those in the FMD group were much more scattered (Fig. 9a). Metabolomics profiles also differed markedly between differently treated mice, with a variety of patterns of changes found between AL and FMD groups. There were considerable overlaps in metabolite profiles observed between the $2 \mathrm{AL}$ groups and $2 \mathrm{FMD}$ groups respectively. Similar to the results of the principal component analysis, distinct patterns were observed when the data were plotted by dendrogram analysis (Fig. 9b). Cluster 1 being more abundant in AL mice and cluster 2 appearing to be more abundant in FMD mice. To obtain a better understanding of the FMD responsible for classification, a sparse partial least squares-discriminant analysis was performed (Fig. 9c). Distinct patterns of AL and FMD mice were observed, and 

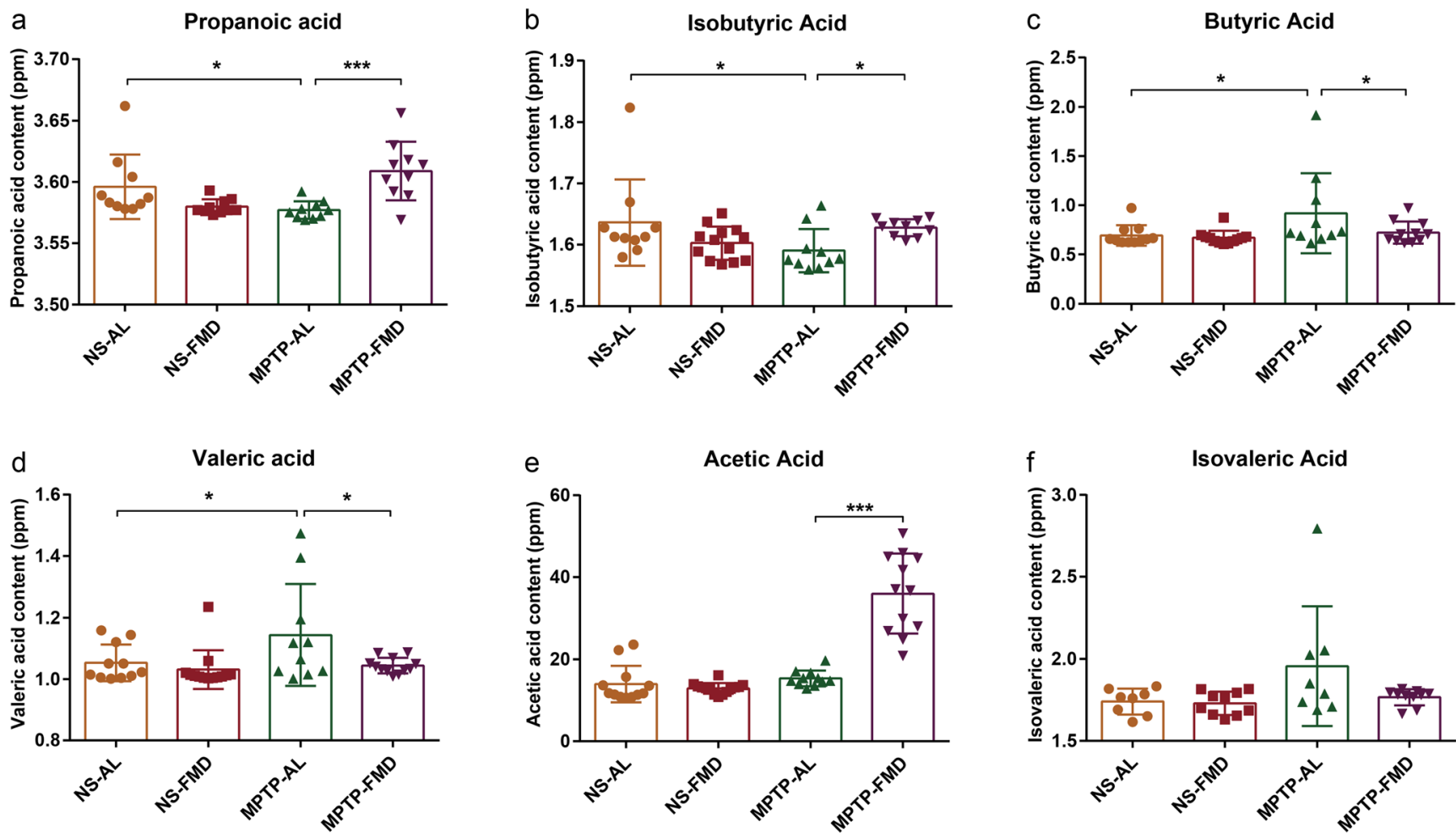

Fig. 8 Fecal SCFAs alterations in mice. (a) Concentration of fecal propionic acid, $F_{3,45}=3.055, p=0.038$. (b) Concentration of fecal isobutyric acid, $F_{3,45}=3.089, p=0.036$. (c) Concentration of fecal butyric acid, $F_{3,45}=3.038, p=0.049$. (d) Concentration of fecal valeric acid, $F_{3,45}=3.072, p=0.037$. (e) Concentration of fecal acetic acid,

patterns of NS-AL (treated with normal saline and ad libitum) and MPTP-AL mice (treated with MPTP and ad libitum) were obviously separated.

\section{Discussion}

Although many studies use fasting as dietary intervention, prolonged water intake only is difficult for the great majority of the population, particularly in old subjects, and its extreme nature could cause the exacerbation of malnourishments and dysfunctions. Therefore, FMD, minimizing the burdens of fasting, will be a better way for studying effects of dietary intervention on neurodegenerative diseases of old people. In the study herein, we report FMD, involving fasting 3 days out of every 7 days over 3 cycles, was found to induce substantial neuroprotective effects in PD mice. FMD mice display significant retention of motor function and inhibition of the loss of dopaminergic neurons in the SN.

In this study, we use PD model by systemic administration of the neurotoxin MPTP to explore the potential role of gut microbiota in FMD-induced neuroprotection in PD. However, according recent studies, MPTP can also produce DAergic selective neuron toxins in the enteric nervous system of animal models, causing damage and pathology in the gastrointestinal
$F_{3,45}=50.632, p<0.001$. (f) Concentration of fecal isovaleric acid, $F_{3,45}=0.952, p=0.424$. Statistical comparison by one-way ANOVA with post hoc comparisons of LSD; data represent the means \pm SEM; ${ }^{*} p<0.05, * * p<0.01, * * * p<0.001$. NS-AL group $(n=12)$, NS-FMD group $(n=14)$, MPTP-AL group $(n=11)$, MPTP-FMD group $(n=12)$

nerves [44-46]. Interestingly, Feng Lai and his colleagues found that MPTP caused gastrointestinal dysfunction and intestinal pathology prior to motor dysfunction, which explains the Braak staging theory that pathogenesis of PD from the gut to the brain [47]. The studies in consistent with this theory demonstrated that $\alpha$-synuclein appears in the enteric nervous system during the early stages of PD, and this misfolded protein may spread to the central nervous system through the vagus nerve, resulting in nerve damage in the $\mathrm{SN}$ [48, 49]. The exact mechanism of MPTP metabolism in brain and gut needs more explorations.

As is well-known, PD is associated with a massive astrogliosis and the presence of microglial cells, which leads to the release of inflammatory cytokines (TNF- $\alpha$ and IL-1 $\beta$ ). In the present study, we show FMD decreases the number of astrocytes and microglial cells in PD mice and prevents the higher levels of TNF- $\alpha$ and IL- $1 \beta$ induced by MPTP. It is known that increases in Clostridium can be a consequence of inflammation [50]. In agreement with study above, Clostridium increase in MPTP-AL mice (treated with MPTP and ad libitum) and retain normal levels in MPTP-FMD mice (treated with MPTP and fasting mimicking diet) in our study, suggesting gut microbiota involved in PD pathogenesis, including inflammation, and FMD prevent the Clostridium dysbiosis. Accumulating evidence highlights that BDNF is a 
Table 1 Metabolites significantly different between NS-AL mice, NS-FMD mice, MPTP-AL mice, and MPTP-FMD mice

\begin{tabular}{|c|c|c|c|c|c|c|}
\hline \multirow[t]{2}{*}{ Metabolite name } & \multicolumn{4}{|l|}{ Group area } & \multicolumn{2}{|l|}{$p$ value } \\
\hline & NS-AL & NS-FMD & MPTP-AL & MPTP-FMD & NA $v s$ MA & MA vs MF \\
\hline Nabilone & $235,989.146$ & $294,765.137$ & $1,257,579.028$ & $215,382.279$ & 0.000 & 0.000 \\
\hline$(+/-)-C 75$ & $680,293.806$ & $517,123.521$ & $1,210,846.783$ & $544,569.239$ & 0.023 & 0.000 \\
\hline DHODA & $327,663.535$ & $532,275.022$ & $877,131.152$ & $462,184.206$ & 0.002 & 0.019 \\
\hline HMD & $53,962.615$ & $62,167.321$ & $86,775.525$ & $68,637.613$ & 0.000 & 0.012 \\
\hline $\mathrm{DD}$ & $100,272.217$ & $148,574.161$ & $484,863.732$ & $153,603.871$ & 0.017 & 0.009 \\
\hline DMTONP & $125,426.346$ & $116,370.042$ & $223,137.245$ & $126,821.000$ & 0.038 & 0.007 \\
\hline DOYOHPG & $25,211.672$ & $12,475.515$ & $45,159.253$ & $20,469.442$ & 0.013 & 0.000 \\
\hline $\mathrm{CB}$ & $145,833.080$ & $154,221.161$ & $271,099.846$ & $159,665.778$ & 0.013 & 0.021 \\
\hline Amiloride & $238,887.600$ & $307,173.260$ & $354,206.744$ & $263,971.306$ & 0.038 & 0.041 \\
\hline Isotretinoin & $59,463.054$ & $60,938.786$ & $127,714.747$ & $47,842.018$ & 0.023 & 0.004 \\
\hline $\mathrm{OA}$ & $1,943,202.465$ & $1,027,283.695$ & $4,580,878.126$ & $671,457.972$ & 0.020 & 0.000 \\
\hline $\mathrm{DA}$ & $480,374.351$ & $431,783.114$ & $1,074,167.337$ & $459,382.734$ & 0.028 & 0.010 \\
\hline Acetylshikonin & $19,175.807$ & $14,936.875$ & $130,986.051$ & $54,539.617$ & 0.000 & 0.000 \\
\hline APM & $4,765,796.770$ & $4,623,011.580$ & $6,367,601.718$ & $4,309,795.694$ & 0.022 & 0.030 \\
\hline Asn-pro & $471,638.531$ & $255,744.091$ & $1,683,488.277$ & $204,730.296$ & 0.029 & 0.000 \\
\hline Butopyronoxyl & $398,110.205$ & $430,235.694$ & $246,370.465$ & $379,338.191$ & 0.009 & 0.002 \\
\hline Azepan-2-one & $603,840.601$ & $1,052,156.329$ & $1,358,330.455$ & $655,218.171$ & 0.019 & 0.031 \\
\hline Capuride & $631,293.088$ & $444,456.466$ & $1,513,175.750$ & $597,204.371$ & 0.014 & 0.012 \\
\hline D-alanyl-D-alanine & $87,004.215$ & $70,330.655$ & $162,157.917$ & $41,723.162$ & 0.040 & 0.000 \\
\hline Dicyclomine & $28,386.440$ & $38,897.550$ & $95,167.637$ & $59,070.153$ & 0.001 & 0.048 \\
\hline Dimetofrine & $1,101,806.320$ & $1,218,218.937$ & $1,552,813.269$ & $1,009,942.181$ & 0.000 & 0.002 \\
\hline DL-glutamine & $963,370.395$ & $852,459.578$ & $1,573,320.886$ & $830,824.823$ & 0.041 & 0.038 \\
\hline Ecgonine & $1,050,773.518$ & $1,155,764.435$ & $1,584,894.876$ & $1,320,973.812$ & 0.003 & 0.022 \\
\hline Fluoxymesterone & $169,478.346$ & $126,366.586$ & $285,577.725$ & $69,871.843$ & 0.021 & 0.000 \\
\hline Gaboxadol & $838,644.723$ & $495,145.180$ & $3,587,141.441$ & $431,456.273$ & 0.001 & 0.000 \\
\hline g-Butyrobetaine & $331,073.074$ & $371,449.711$ & $143,574.251$ & $264,044.660$ & 0.003 & 0.026 \\
\hline Imazapyr & $356,735.134$ & $292,676.658$ & $535,542.221$ & $343,288.343$ & 0.048 & 0.016 \\
\hline Kanosamine & $279,368.544$ & $291,453.904$ & $674,447.531$ & $258,624.473$ & 0.025 & 0.006 \\
\hline L-alanyl-L-proline & $455,340.888$ & $598,910.371$ & $2,976,694.869$ & $368,637.531$ & 0.000 & 0.000 \\
\hline L-alanyl-L-proline & $89,073.879$ & $53,265.814$ & $205,415.316$ & $82,780.940$ & 0.044 & 0.001 \\
\hline $\mathrm{CHL}$ & $55,659.106$ & $57,587.451$ & $92,877.573$ & $39,122.642$ & 0.023 & 0.000 \\
\hline TBL & $289,761.462$ & $323,549.493$ & $102,525.415$ & $423,331.316$ & 0.003 & 0.000 \\
\hline DT & $434,040.911$ & $426,685.688$ & $782,288.765$ & $429,381.221$ & 0.041 & 0.007 \\
\hline $\mathrm{AG}$ & $563,300.118$ & $484,839.231$ & $1,438,941.847$ & $320,271.507$ & 0.006 & 0.000 \\
\hline $\mathrm{N}$-acetyl-L-histidine & $472,083.471$ & $318,819.989$ & $1,027,780.111$ & $430,988.660$ & 0.042 & 0.026 \\
\hline $\mathrm{OM}$ & $614,273.359$ & $577,943.473$ & $449,549.780$ & $663,925.220$ & 0.012 & 0.044 \\
\hline Oxaceprol & $252,224.047$ & $290,325.254$ & $535,997.134$ & $293,632.530$ & 0.001 & 0.001 \\
\hline Oxaceprol & $530,732.549$ & $306,802.171$ & $849,670.647$ & $398,505.474$ & 0.023 & 0.000 \\
\hline $\mathrm{PE}$ & $1,075,226.380$ & $1,063,171.498$ & $2,396,963.022$ & $979,733.677$ & 0.008 & 0.000 \\
\hline Acetamide & $49,648.898$ & $47,154.069$ & $125,139.271$ & $42,930.012$ & 0.005 & 0.004 \\
\hline QV1MVO1R & $508,324.698$ & $648,284.260$ & $833,894.039$ & $582,818.321$ & 0.000 & 0.004 \\
\hline Ricinoleic acid & $1,332,037.285$ & $1,903,531.420$ & $2,847,023.712$ & $1,188,492.912$ & 0.002 & 0.000 \\
\hline Ricinoleic acid & $246,590.512$ & $241,407.359$ & $572,062.309$ & $267,292.771$ & 0.012 & 0.023 \\
\hline trans-Anethole & $58,787.265$ & $66,823.862$ & $206,866.881$ & $135,176.683$ & 0.000 & 0.020 \\
\hline UNII:OUT5YHB7BO & $87,853.504$ & $99,590.773$ & $166,246.580$ & $82,988.887$ & 0.026 & 0.000 \\
\hline
\end{tabular}

In all metabolites mentioned above, there is no significant difference of group area between NA group and NF group $(p \geq 0.05)$. Statistical comparison by one-way ANOVA with post hoc comparisons of LSD

NA = NS-AL group $(n=12)$; NF = NS-FMD group $(n=18)$; MA = MPTP-AL group $(n=14) ; \mathrm{MF}=\mathrm{MPTP}-\mathrm{FMD}$ group $(n=16) ;$ DHODA = 5Z,11 beta,13E,15S)-11,15-dihydroxy-N-(2-hydroxyethyl)-9-oxoprosta-5,13-dien-1-amide; HMD = 1-(4-hydroxy-3-methoxyphenyl)-3,5diacetoxyoctane; DD = 1,1'-[1,12-dodecanediylbis(oxy)]dibenzene; DMTONP = 1,2-dideoxy-3-C-methyl-1-(2,5,5,8a-tetramethyl-1,4,4a,5,6,7,8,8aoctahydro-1-naphthalenyl)pentitol; DOYOHPG = 1-O-[\{[(2E)-3,7-dimethyl-2,6-octadien-1-yl]oxy $\}$ (hydroxy)phosphoryl]-beta-D-glucopyranose

key protein in brain plasticity and is particularly important for survival of dopaminergic neurons [51-53]. BDNF is not only expressed by neurons but is also found in astrocytes [54] and microglia $[55,56]$. Microglial BDNF was first shown in microglia cultures $[57,58]$ and soon confirmed in different regions of the central nervous system during the course of various neurological disorders, including Parkinson's disease
Fig. 9 Metabolic profiles of fecal samples from the four groups of mice. (a) Plots of principal component analysis scores of fecal samples derived from the liquid chromatography-mass spectrometry metabolite profiles. (b) Hierarchical clustering analysis of fecal samples. (c) Sparse partial least squares-discriminant analysis score plots of fecal samples derived from the liquid chromatography-mass spectrometry metabolite profiles. $\mathrm{NA}=\mathrm{NS}-\mathrm{AL}$ group $(n=12)$; MA = MPTP-AL group $(n=14)$; NF = NSFMD group $(n=18)$; MF $=$ MPTP-FMD group $(n=16)$ 

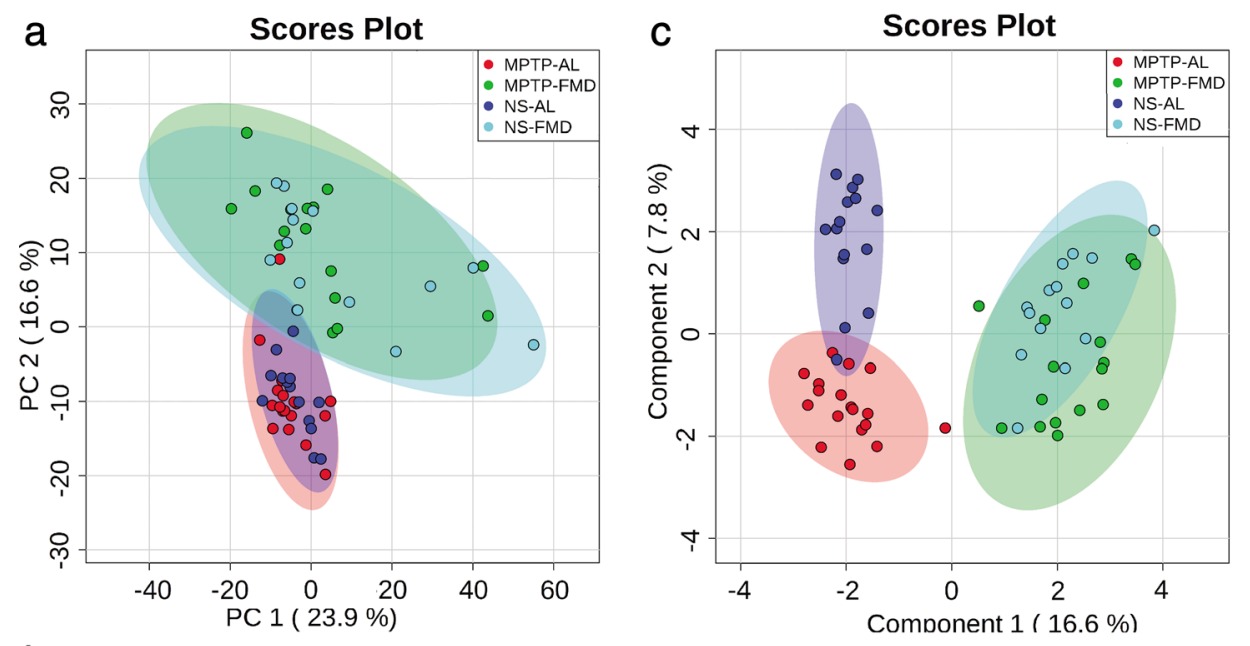

b

- MPTP-AL

- MPTP-FMD

- NS-AL

- NS-FMD

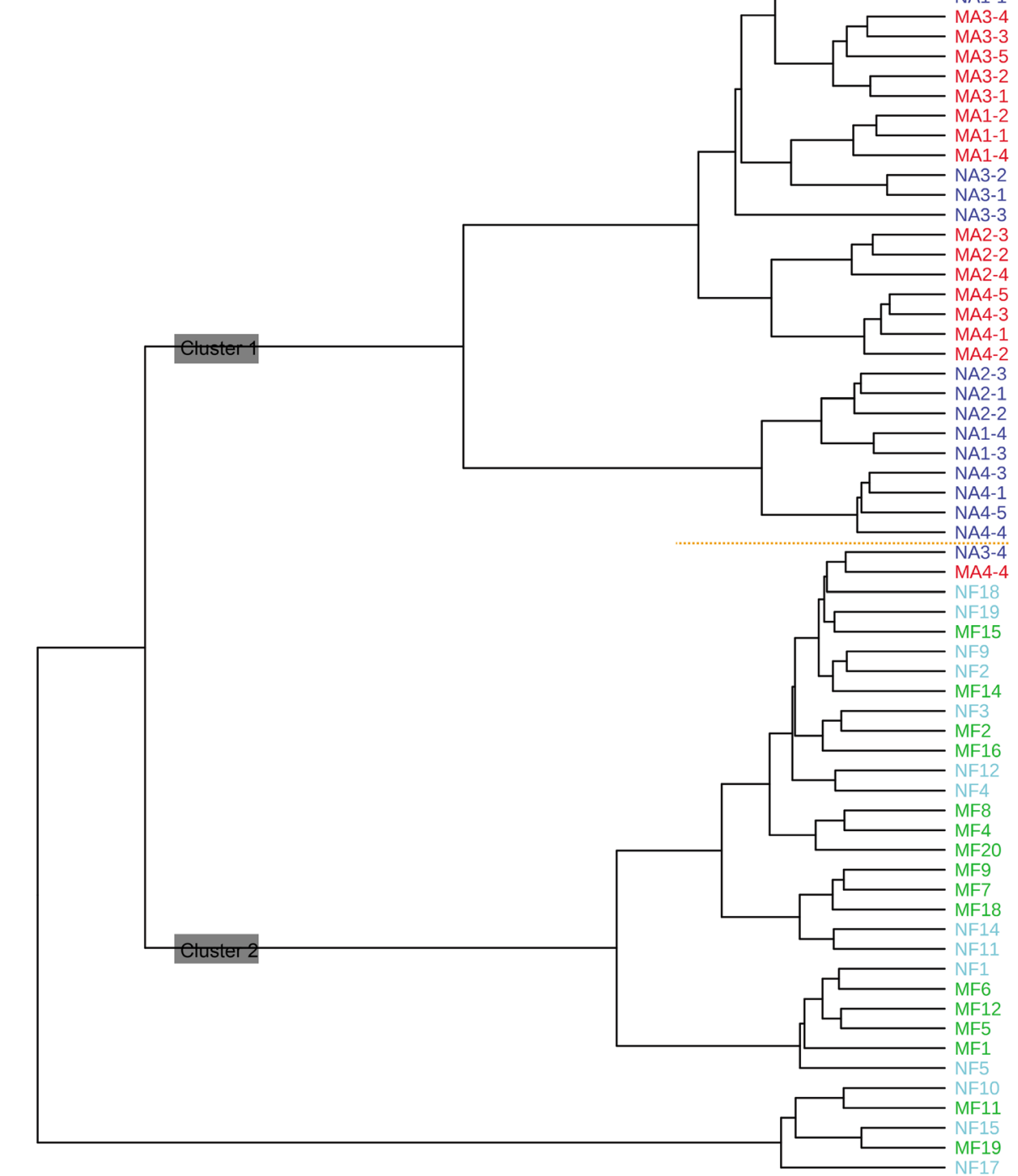

\begin{tabular}{|cccccc}
\hline 250 & 1 & 1 & 1 & \\
200 & 150 & 100 & 50 & 0
\end{tabular}


[59]. The activated microglia and astrocytes can release proinflammatory cytokines such as IL- $1 \beta$ and TNF- $\alpha$, which can aggravate and propagate neuro-inflammation. IL- $1 \beta$ has been demonstrated to block induction of BDNF [60], which is a critical player in normal neurodevelopment and is a neurotrophin that supports neuronal survival and growth. Tong and colleagues found that IL- $1 \beta$ can compromise neuronal survival by interfering with the neuroprotective effect of BDNF [61]. In our study, FMD significantly decreases the number of microglia and astrocytes in PD mice and prevents the release of TNF- $\alpha$ and IL- $1 \beta$ induced by MPTP, which can alleviate neuroinflammation. FMD also inhibits the MPTP-induced decrease in brain BDNF. Combined previous reports and our results, we raised an inference that FMD treatment possibly retain the balance of brain glia and BDNF in PD mice via inhibition of neuroinflammation to protect dopaminergic neurons. Consistently, previous studies showed that caloric restriction restores the level of BDNF to protect neurons against excitotoxicity [7, 62]. Also, intermittent fasting ameliorates cognitive deficits in a rat model of sepsis by suppression of the expression of pro-inflammatory cytokines (TNF- $\alpha$ and IL-1 $\beta$ ) and enhancement of neurotrophic support (BDNF levels) in the hippocampus [63].

A striking finding from this study is that gut microbiota contributes to the FMD-induced neuroprotection in PD. We and others have demonstrated that gut microbial dysbiosis occurs in PD patients and animal models $[20,64]$ and extend these studies to show that alterations of gut microbiota composition may also contribute to PD pathogenesis. In the present study, bacteria belonging to the genus Blautia is significantly reduced in MPTP-AL mice as compared with NS-AL mice, which has been reported that it reduces significantly in feces of PD patients as compared to controls [65]. Intriguingly, FMD can modulate the gut microbiota composition to improve $\mathrm{PD}$, including suppressing the decline of striatal DA and 5-HT levels induced by MPTP, through alleviation of microbial dysbiosis in our study. The serotonergic system is known to be critical in behavior that involves a high cognitive demand [66]. 5-HT functions as a key neurotransmitter at both terminals of gut-brain axis network. It may be related to the ability of the gut microbiota to increase the production of neuroactive metabolites [67]. Simultaneously, microbiota can influence central nervous system serotonergic neurotransmission through a humoral route [68]. Furthermore, microbiota transplantation from mice, on an FMD regimen, into antibiotic-pretreated mice was able to confer neuroprotection, including reducing the MPTP-mediated decreases in striatal DA and 5-HT. This finding not only strongly supports that the beneficial effects of FMD may be mediated by the gut microbiome but also suggests that the gut microbiota is the mediator for the diet-gut-brain axis.

SCFAs, including acetic acid, propionic acid, butyric acid, isobutyric acid, valeric acid, and isovaleric acid, are end products from intestinal microbial fermentation. Recently, the involvement of SCFAs in PD pathogenesis and neuroprotection has been extensively studied. In this study, higher concentrations of butyric acid in MPTP-AL mice might result from the increased OTU abundance of Ruminococcus, which is a butyrate producer [69]. Our results are consistent with another study [70] showing concentrations of valeric acid are significantly higher in patients with stroke compared with the controls and are positively correlated with levels of inflammatory markers. So, it is reasonable to infer that higher levels of valeric acid in MPTP-AL mice are caused by neuro-inflammation induced by MPTP, and FMD restores the level of valeric acid via inhibition of neuro-inflammation by regaining the balance of glia and BDNF in PD mice. However, alterations of fecal SCFAs in PD are not always consistent. Unger et al. [71] found that fecal SCFA concentrations are significantly reduced in PD patients. Intriguingly, change of propionic acid levels in PD mice is different from that we reported previously [64], which may result from different dose of MPTP. Furthermore, we conducted preliminary study of untargeted metabolomics to give a general outline of metabolite changes, among which 45 significant differential metabolites are identified.

In conclusion, the present work offers novel perspectives on a therapeutic approach for the treatment of PD. FMD can recover the motor function, alleviate the loss of dopaminergic neurons in the substantia nigra pars compacta, and increase the levels of DA and 5-HT in the striatum of PD mice, probably by reshaping gut microbiota to modulate microbial dysbiosis, which would lead to changes in SCFAs, suggesting that FMD-induced neuroprotection in PD mice is mediated by a diet-gut-microbiota-brain axis, at least one of the possible pathways. The imbalance of glial cells and neurotrophic factors in PD mice may be also alleviated by FMD via inhibition of neuro-inflammatory. However, screening of functional gut microbiota and metabolites, pathway analysis of metabolites and multilevel analysis are required for the exact mechanism.

Acknowledgments This study was supported by National Natural Science Foundation of China $(81771384,81801276)$, Postgraduate Research \& Practice Innovation (KYCX18_1870), Public Health Research Center at Jiangnan University (JUPH 201801 ), and national first-class discipline program of Food Science and Technology (JUFSTR20180101). We sincerely thank Dr. Stanley Li Lin's careful revision on the manuscript.

Required Author Forms Disclosure forms provided by the authors are available with the online version of this article.

Publisher's Note Springer Nature remains neutral with regard to jurisdictional claims in published maps and institutional affiliations.

\section{References}

1. Poewe W, Seppi K, Tanner CM, et al. Parkinson disease. Nature Reviews Disease Primers 2017; 3: 17013. 
2. Cacabelos R. Parkinson's disease: from pathogenesis to pharmacogenomics. International Journal of Molecular Sciences 2017; 183: 551 .

3. Mukherjee A, Biswas A, and Das SK. Gut dysfunction in Parkinson's disease. World Journal of Gastroenterology 2016; 2225: 5742-5752.

4. Thomas B and Beal MF. Parkinson's disease. Hum Mol Genet 2007; 16 Spec No. 2: 183-194.

5. Bousquet M, St-Amour I, Vandal M, et al. High-fat diet exacerbates MPTP-induced dopaminergic degeneration in mice. Neurobiology of Disease 2012; 451: 529-538.

6. Choi JY, Jang EH, Park CS, and Kang JH. Enhanced susceptibility to 1-methyl-4-phenyl-1,2,3,6-tetrahydropyridine neurotoxicity in high-fat diet-induced obesity. Free Radic Biol Med 2005; 386: 806-816.

7. Maswood N, Young J, Tilmont E, et al. Caloric restriction increases neurotrophic factor levels and attenuates neurochemical and behavioral deficits in a primate model of Parkinson's disease. Proc Natl Acad Sci U S A 2004; 10152: 18171-18176.

8. Duan WZ, Guo ZH, Jiang HY, et al. Dietary restriction normalizes glucose metabolism and BDNF levels, slows disease progression, and increases survival in huntingtin mutant mice. Proc Natl Acad Sci U S A 2003; 1005: 2911-2916.

9. Choi IY, Piccio L, Childress P, et al. A diet mimicking fasting promotes regeneration and reduces autoimmunity and multiple sclerosis symptoms. Cell Rep 2016; 1510: 2136-2146.

10. Brandhorst $\mathrm{S}$, Choi IY, Wei M, et al. A periodic diet that mimics fasting promotes multi-system regeneration, enhanced cognitive performance, and healthspan. Cell Metab 2015; 221: 86-99.

11. Wei M, Brandhorst S, Shelehchi M, et al. Fasting-mimicking diet and markers/risk factors for aging, diabetes, cancer, and cardiovascular disease. Sci Transl Med 2017; 9377.

12. Cheng CW, Villani V, Buono R, et al. Fasting-mimicking diet promotes Ngn3-driven beta-cell regeneration to reverse diabetes. Cell 2017; 1685: 775-788.

13. Deitch EA, Winterton J, and Berg R. Effect of starvation, malnutrition, and trauma on the gastrointestinal tract flora and bacterial translocation. Arch Surg 1987; 1229: 1019-1024.

14. Sonoyama K, Fujiwara R, Takemura N, et al. Response of gut microbiota to fasting and hibernation in Syrian hamsters. Appl Environ Microbiol 2009; 7520: 6451-6456.

15. Li GL, Xie C, Lu SY, et al. Intermittent fasting promotes white adipose browning and decreases obesity by shaping the gut microbiota. Cell Metab 2017; 264: 672-685.

16. Zarrinpar A, Chaix A, Yooseph S, and Panda S. Diet and feeding pattern affect the diurnal dynamics of the gut microbiome. Cell Metab 2014; 206: 1006-1017.

17. Zhang LS and Davies SS. Microbial metabolism of dietary components to bioactive metabolites: opportunities for new therapeutic interventions. Genome Med 2016; 8: 46.

18. Luczynski P, Neufeld KAM, Oriach CS, et al. Growing up in a bubble: using germ-free animals to assess the influence of the gut microbiota on brain and behavior. Int J Neuropsychopharmacol 2016; 198: 1-17.

19. Sampson TR, Debelius JW, Thron T, et al. Gut microbiota regulate motor deficits and neuroinflammation in a model of Parkinson's disease. Cell 2016; 1676: 1469-1480.

20. Qian Y, Yang X, Xu S, et al. Alteration of the fecal microbiota in Chinese patients with Parkinson's disease. Brain Behav Immun 2018; 70: 194-202.

21. Yang X, Qian Y, Xu S, Song Y, and Xiao Q. Longitudinal analysis of fecal microbiome and pathologic processes in a rotenone induced mice model of Parkinson's disease. Front Aging Neurosci 2017; 9: 441.

22. Torres ERS, Akinyeke T, Stagaman K, et al. Effects of sub-chronic MPTP exposure on behavioral and cognitive performance and the microbiome of wild-type and mGlu8 knockout female and male mice. Front Behav Neurosci 2018; 12: 140.

23. Clarke TB, Davis KM, Lysenko ES, et al. Recognition of peptidoglycan from the microbiota by Nod1 enhances systemic innate immunity. Nat Med 2010; 162: 228-231.

24. Schuijt TJ, Lankelma JM, Scicluna BP, et al. The gut microbiota plays a protective role in the host defence against pneumococcal pneumonia. Gut 2016; 654: 575-583.

25. Chen GY, Shaw MH, Redondo G, and Nunez G. The innate immune receptor Nod1 protects the intestine from inflammationinduced tumorigenesis. Cancer Res 2008; 6824: 10060-10067.

26. Gacias M, Gaspari S, Santos PMG, et al. Microbiota-driven transcriptional changes in prefrontal cortex override genetic differences in social behavior. Elife 2016; 5: e13442.

27. Ricaurte GA, Langston JW, Delanney LE, et al. Fate of nigrostriatal neurons in young mature mice given 1-methyl-4-phenyl-1,2,3,6tetrahydropyridine: a neurochemical and morphological reassessment. Brain Res 1986; 3761: 117-124.

28. Kiriyama K, Ohtaki H, Kobayashi N, et al. A nucleoproteinenriched diet suppresses dopaminergic neuronal cell loss and motor deficit in mice with MPTP-induced Parkinson's disease. J Mol Neurosci 2015; 553: 803-811.

29. Ubeda C, Bucci V, Caballero S, et al. Intestinal microbiota containing barnesiella species cures vancomycin-resistant Enterococcus faecium colonization. Infect Immun 2013; 813: 965-973.

30. Cao Q, Qin LY, Huang F, et al. Amentoflavone protects dopaminergic neurons in MPTP-induced Parkinson's disease model mice through PI3K/Akt and ERK signaling pathways. Toxicol Appl Pharmacol 2017; 319: 80-90.

31. $\mathrm{Hu} \mathrm{M}, \mathrm{Li} \mathrm{FM}$, and Wang WD. Vitexin protects dopaminergic neurons in MPTP-induced Parkinson's disease through PI3K/Akt signaling pathway. Drug Design Development and Therapy 2018; 12 : 565-573.

32. Ahmed M and Ghanem A. Enantioselective nano liquid chromatographic separation of racemic pharmaceuticals: a facile one-pot in situ preparation of lipase-based polymer monoliths in capillary format. Chirality 2014; 2611: 754-763.

33. Peiffer JA, Spor A, Koren O, et al. Diversity and heritability of the maize rhizosphere microbiome under field conditions. Proc Natl Acad Sci U S A 2013; 11016: 6548-6553.

34. Baxter NT, Wan JJ, Schubert AM, et al. Intra- and interindividual variations mask interspecies variation in the microbiota of sympatric peromyscus populations. Appl Environ Microbiol 2015; 811: 396-404.

35. Caporaso JG, Kuczynski J, Stombaugh J, et al. QIIME allows analysis of high-throughput community sequencing data. Nat Methods 2010; 75: 335-336.

36. Edgar RC. Search and clustering orders of magnitude faster than BLAST. Bioinformatics 2010; 2619: 2460-2461.

37. DeSantis TZ, Hugenholtz P, Larsen N, et al. Greengenes, a chimerachecked 16S rRNA gene database and workbench compatible with ARB. Appl Environ Microbiol 2006; 727: 5069-5072.

38. Wang Q, Garrity GM, Tiedje JM, and Cole JR. Naive Bayesian classifier for rapid assignment of rRNA sequences into the new bacterial taxonomy. Appl Environ Microbiol 2007; 7316: 52615267.

39. Garcia-Villalba R, Gimenez-Bastida JA, Garcia-Conesa MT, et al. Alternative method for gas chromatography-mass spectrometry analysis of short-chain fatty acids in faecal samples. J Sep Sci 2012; 3515: 1906-1913.

40. Zhao L, Zhang F, Ding X, et al. Gut bacteria selectively promoted by dietary fibers alleviate type 2 diabetes. Science $2018 ; 3596380$ : $1151-1156$.

41. Xu W, Chen D, Wang N, et al. Development of high-performance chemical isotope labeling LC-MS for profiling the human fecal metabolome. Anal Chem 2015; 872: 829-836. 
42. Palasz E, Bak A, Gasiorowska A, and Niewiadomska G. The role of trophic factors and inflammatory processes in physical activityinduced neuroprotection in Parkinson's disease. Postepy Hig Med Dosw (Online) 2017; 711: 713-726.

43. Lei E, Vacy K, and Boon WC. Fatty acids and their therapeutic potential in neurological disorders. Neurochem Int 2016; 95: 7584.

44. Anderson G, Noorian AR, Taylor G, et al. Loss of enteric dopaminergic neurons and associated changes in colon motility in an MPTP mouse model of Parkinson's disease. Exp Neurol 2007; 2071: 4-12.

45. Natale G, Kastsiushenka O, Fulceri F, et al. MPTP-induced parkinsonism extends to a subclass of TH-positive neurons in the gut. Brain Res 2010; 1355: 195-206.

46. Cote M, Drouin-Ouellet J, Cicchetti F, and Soulet D. The critical role of the MyD88-dependent pathway in non-CNS MPTP-mediated toxicity. Brain Behav Immun 2011; 256: 1143-1152.

47. Lai F, Jiang R, Xie W, et al. Intestinal pathology and gut microbiota alterations in a methyl-4-phenyl-1,2,3,6-tetrahydropyridine (MPTP) mouse model of Parkinson's disease. Neurochem Res 2018; 4310: 1986-1999.

48. Braak H, de Vos RA, Bohl J, and Del Tredici K. Gastric alphasynuclein immunoreactive inclusions in Meissner's and Auerbach's plexuses in cases staged for Parkinson's diseaserelated brain pathology. Neurosci Lett 2006; 3961: 67-72.

49. Phillips RJ, Walter GC, Wilder SL, Baronowsky EA, and Powley TL. Alpha-synuclein-immunopositive myenteric neurons and vagal preganglionic terminals: autonomic pathway implicated in Parkinson's disease? Neuroscience 2008; 1533: 733-750.

50. Scheperjans F, Aho V, Pereira PAB, et al. Gut microbiota are related to Parkinson's disease and clinical phenotype. Mov Disord 2015; 303: 350-358.

51. Howells DW, Porritt MJ, Wong JY, et al. Reduced BDNF mRNA expression in the Parkinson's disease substantia nigra. Exp Neurol 2000; 1661: 127-135.

52. Baquet ZC, Gorski JA, and Jones KR. Early striatal dendrite deficits followed by neuron loss with advanced age in the absence of anterograde cortical brain-derived neurotrophic factor. J Neurosci 2004; 2417: 4250-4258.

53. Baydyuk M, Nguyen MT, and Xu B. Chronic deprivation of TrkB signaling leads to selective late-onset nigrostriatal dopaminergic degeneration. Exp Neurol 2011; 2281: 118-125.

54. Bergami M, Santi S, Formaggio E, et al. Uptake and recycling of pro-BDNF for transmitter-induced secretion by cortical astrocytes. J Cell Biol 2008; 1832: 213-221.

55. Coull JA, Beggs $\mathrm{S}$, Boudreau D, et al. BDNF from microglia causes the shift in neuronal anion gradient underlying neuropathic pain. Nature 2005; 4387070: 1017-1021

56. Parkhurst CN, Yang G, Ninan I, et al. Microglia promote learningdependent synapse formation through brain-derived neurotrophic factor. Cell 2013; 1557: 1596-1609.
57. Elkabes S, DiCicco-Bloom EM, and Black IB. Brain microglia/ macrophages express neurotrophins that selectively regulate microglial proliferation and function. J Neurosci 1996; 168: 2508-2521.

58. Miwa T, Furukawa S, Nakajima K, Furukawa Y, and Kohsaka S. Lipopolysaccharide enhances synthesis of brain-derived neurotrophic factor in cultured rat microglia. J Neurosci Res 1997; 506: 1023-1029.

59. Knott C, Stern G, Kingsbury A, Welcher AA, and Wilkin GP. Elevated glial brain-derived neurotrophic factor in Parkinson's diseased nigra. Parkinsonism Relat Disord 2002; 85: 329-341.

60. Barrientos RM, Sprunger DB, Campeau S, et al. BDNF mRNA expression in rat hippocampus following contextual learning is blocked by intrahippocampal IL-1beta administration. J Neuroimmunol 2004; 1551-2: 119-126.

61. Tong L, Balazs R, Soiampornkul R, Thangnipon W, and Cotman CW. Interleukin-1 beta impairs brain derived neurotrophic factorinduced signal transduction. Neurobiol Aging 2008; 299: 13801393.

62. Duan W, Guo Z, and Mattson MP. Brain-derived neurotrophic factor mediates an excitoprotective effect of dietary restriction in mice. J Neurochem 2001; 762: 619-626.

63. Vasconcelos AR, Yshii LM, Viel TA, et al. Intermittent fasting attenuates lipopolysaccharide-induced neuroinflammation and memory impairment. J Neuroinflammation 2014; 11: 85.

64. Sun MF, Zhu YL, Zhou ZL, et al. Neuroprotective effects of fecal microbiota transplantation on MPTP-induced Parkinson's disease mice: gut microbiota, glial reaction and TLR4/TNF-alpha signaling pathway. Brain Behav Immun 2018; 70: 48-60.

65. Keshavarzian A, Green SJ, Engen PA, et al. Colonic bacterial composition in Parkinson's disease. Mov Disord 2015; 3010: 13511360.

66. Jenkins TA, Nguyen JC, Polglaze KE, and Bertrand PP. Influence of tryptophan and serotonin on mood and cognition with a possible role of the gut-brain axis. Nutrients 2016; 81: E56.

67. O'Mahony SM, Clarke G, Borre YE, Dinan TG, and Cryan JF. Serotonin, tryptophan metabolism and the brain-gut-microbiome axis. Behav Brain Res 2015; 277: 32-48.

68. Clarke G, Grenham S, Scully P, et al. The microbiome-gut-brain axis during early life regulates the hippocampal serotonergic system in a sex-dependent manner. Mol Psychiatry 2013; 186: 666-673.

69. Westfall S, Lomis N, Kahouli I, et al. Microbiome, probiotics and neurodegenerative diseases: deciphering the gut brain axis. Cell Mol Life Sci 2017; 7420: 3769-3787.

70. Yamashiro K, Tanaka R, Urabe T, et al. Gut dysbiosis is associated with metabolism and systemic inflammation in patients with ischemic stroke. PLoS One 2017; 122: e0171521.

71. Unger MM, Spiegel J, Dillmann KU, et al. Short chain fatty acids and gut microbiota differ between patients with Parkinson's disease and age-matched controls. Parkinsonism Relat Disord 2016; 32: 66-72. 